\title{
The Intertwining Impact of Intraorganizational and Routine Networks on Routine Replication Dynamics: An Agent-Based Model
}

\author{
Dehua Gao $\mathbb{D D}^{1}{ }^{1}$ Flaminio Squazzoni, ${ }^{2}$ and Xiuquan Deng ${ }^{3}$ \\ ${ }^{1}$ School of Management Science and Engineering, Shandong Technology and Business University, Yantai 264005, China \\ ${ }^{2}$ Department of Economics and Management, University of Brescia, Brescia 25122, Italy \\ ${ }^{3}$ School of Economics and Management, Beihang University, Beijing 100191, China \\ Correspondence should be addressed to Dehua Gao; dhuagao@gmail.com
}

Received 8 April 2018; Revised 2 October 2018; Accepted 15 October 2018; Published 11 November 2018

Academic Editor: Yan-Ling Wei

Copyright (C) 2018 Dehua Gao et al. This is an open access article distributed under the Creative Commons Attribution License, which permits unrestricted use, distribution, and reproduction in any medium, provided the original work is properly cited.

\begin{abstract}
Organizational routines are means through which organizations can reutilize best practices and so their replication, i.e., duplicating beneficial routines across context, is a key value-creating strategy. However, it is difficult to map network effects on routine replication. Here, we investigated routine replicating dynamics considering two types of network contexts, namely, (1) connections between different (geographically distributed) units in a decentralized organization and (2) the coupling relation between routines, i.e., a bundle of different routines involved in each unit. By considering routine replication as one kind of template-based activities between different units, we examined interrelations between routines with a NK-based fitness landscape model. Our results show that when there is an appropriate level of absorptive capacities (i.e., when organizations are capable of identifying and acquiring externally generated knowledge), there is an optimal combination of these two types of networks, which is beneficial to routine replicating practices and organization adaptation. Furthermore, we also found that intraorganizational variations, including template-duplicating errors and innovative activities, are instrumental to enhance adaptive changes. Our findings suggest measures to control and manage best practice diffusion across organizations.
\end{abstract}

\section{Introduction}

Recent research has shown that organizational routines, i.e., "repetitive, recognizable pattern of interdependent actions" carried out by multiple individual actors [1], are "ubiquitous" in almost every organization [2]. While certain scholars have focused on the so-called "defensive routines", i.e., actions or policies that "prevent either individuals or segments of the organization from experiencing embarrassment or threat" $[3,4]$, and explored their negative effect on organizational learning and change [5-7], there is evidence that organizational routines are a key repository of organizational memory, skills and tacit knowledge which enables both organizational stability and change [1, 8-11] and constitutes organizations' sustained competitive advantages [12-14]. In this sense, by applying routines representing "best practices" to new environments, organizational core capabilities can be enhanced that in turn further sustain competitive advantages and profitability [15]. This is why the replication of organizational routines is considered as an important value-creating strategy and attracted more increasing attention from both researchers and practitioners [16-18].

Firesl and Larty [18] summarized three value-creating mechanisms of replicating routines for which replication is viewed (1) as a strategy for growth; (2) a means to create value by improving organizational efficiency; and (3) an organizational scaffold that may enhance flexibility. These value-creating mechanisms, according to Jenson [19] and D’Adderio [20], are particularly applicable to organizations, such as large or multinational firms, within which multiple similar units perform the same function, or franchise and chain stores, which imply the geographic replication of similar units. 
According to Szulanski and Jensen [21], replicating a routine involves the creation of another routine that is 'similar to the original routine in significant respects.' However, Howard-Grenville [22] pointed out that organizational routines might perform differently depending on specific organizational environments. Given certain context-dependent characteristics, the replication of organizational routines can be understood as a simple knowledge transfer process. Indeed, any match between these newly created routines and the context they were originally coping with imposes what Winter and Szulanski [15] called a "replication dilemma" problem. This means that besides the exact "cloning" of the original routines, these new routines must be to some extent flexible and adaptive to be suitable to the new, in part similar but also different, organizational context $[18,20]$.

Therefore, replication of routines is a means through which organizations can reutilize knowledge that is already in use [21, 23]. Some scholars focused on the importance of replication as a "knowledge transfer" across intraorganizational boundaries [23-26] and considered the intraorganizational transferring process between different units, often across different geographic locations, from a network-based perspective [27-29]. Here, there is the important role of templates during the replicating process of routines $[21,30]$ as a means to stimulate forward and reverse knowledge flows [18].

As suggested by D'Adderio [20] and Friesl and Larty [31], replicating routines is a complex social practice, which involves multiple actors and artifacts. For instance, the capacity of individual actors to understand specific routines is always intertwined with their own behaviors, experiences, and the particular environment within which they live [12]. Therefore, it is likely that individuals have to make contextspecific adjustments so as to reproduce "the similar routine" in prospection [11,32-34]. Unfortunately, as suggested by Friesl and Larty [18, 31], we still know little about the micro processes through which individual actors and actions shape and enact new routines in their new contexts.

Furthermore, research on organizational routines has emphasized the connective nature of routines in that organizations and capabilities are better understood as dependent on some "specific networks of routines," rather than just on bundles of them [11,35-37]. This is because routines often do not work alone but are interrelated with each other [38]. Secondly, recent findings indicated that the dynamics of several interrelated routines together, which are often what occurs in practice and to some extent determines organizational outcomes, are definitely different from that of individual routines [39]. If so, routine interdependency can be a fundamental source of both organizational stability and flexibility [40]. However, as recently suggested by Feldman et al. [11] and Kremser and Schreyögg [39], more research is needed to understand 'implications and consequences of routine interrelatedness.'

Our paper aims to investigate the replicating dynamics of organizational routines within two types of network contexts. First, we hypothesized connections between (geographically) distributed units of an organization [18, 41, 42]. In a second scenario, we hypothesized a routine-related network - i.e., a network of different routines involved in the whole organization [11]. To do so, we first followed the NK and fitness landscape modeling [43] to depict routine networks and built up the fitness landscape of an organization. Then, we translated this into an agent-based model (from now on, $\mathrm{ABM}$ ) [44-46] to explore complex behaviors of routine replications.

In our model, we focused neither on individual actors nor on artifacts in the enactment of routines in new contexts. Rather, we treated an aggregation of these individuals and artifacts, namely, the unit distributed within an organization, which we called a "unit", as the basic heterogeneous but interdependent agent. All the units (i.e., agents) randomly interacted with each other, while by using templates specific routines were replicated from unit to unit. Furthermore, we also considered the impact of both internal variability and external dynamics of the organizational environment on routine replications. This included, for example, templateduplicating errors and innovative activities among units and the pace of environmental changes.

The rest of the paper is structured as follows. In the second section, we provide a brief literature overview on replication of organizational routines. Further, we also summarized the feasibility and effectiveness of adopting an NK-inspired ABM to examine organizational routines. Then, we present a series of concrete scenarios with combinations of input settings and show our simulation results. Finally, the last section summarizes our main findings, discusses limitations, and elaborates on implications.

\section{Theoretical Foundations}

2.1. Replication of Routines within Organizations. Nelson and Winter [8] and Zollo and Winter [47] considered an organization as "a set of interdependent operational and administrative routines." These different kinds of routines embody an important part of the organization's productive knowledge [8] and are considered as one of the key sources for sustained competitive advantages [12, 48, 49]. This suggests that sustaining competitive advantages can also depend on the capability of replicating the most efficacious routines from a unit to another, or even from an organization to another, just as efficacious habits are replicated from an individual to another one $[17,50]$. However, in some respects, routine replication can be more difficult than the replication of individual habits. First, after discovering an efficacious practice, managers tend to build organizational barriers against replication, so as to exploit the system's advantage and avoid imitation by others [51]. Second, knowledge embedded in routines is always to some extent not explicit but tacit and enacted in practice $[32,47]$. At the same time, either units or organizations have only limited capacities to discern superior practices from their neighbors [50].

Furthermore, given the "context-dependent" characteristics of routines [22] and the need for adaptive changes [15, 52], for any organization, routine replication has value when it can support appropriate variations that are instrumental to cope with environmental challenges $[15,18,20]$. 
In this respect, by discussing the connection between intraorganizational replication and interorganizational imitation, Rivkin [51] further pinpointed that replication can be more efficient than imitation in promoting sustained competitive advantage only when the complexity of the task and the environment is moderate. Winter and Szulanski [23] distinguished the term "replication" from similar concepts such as "transfer of knowledge" and "diffusion of innovations" within and/or even between organizations. They suggested that replication involves the creation of "a series of local routines that are quite similar to the original ones in significant respects" [23]. This implies that replication often relates to an organization's attempts to "reproduce at multiple internal sites the outcome of an existing activity" and so "applies to a broad class of exploitation situations" [8,23].

Since Nelson and Winter [8], many scholars explored the dynamics of replication, such as routine replication barriers $[24,53]$, the impact of replicator/replicatee relationship, and the role of institutional contexts [54]. Others looked at the movement of employees or independent experts as a carrier of knowledge and experience transfer from one context to another (e.g., Hodgson [50]). If replication is a "knowledge transferring" process across intraorganizational boundaries $[24,25]$, this process depends on the fact that the 'core aspects of a routine', or even "a whole business format", can be truly communicated from one unit to another, which then transforms related knowledge into practice [18].

Research suggested that "templates" are key for replicating organizational routines [21, 52]. Here, the term "template," according to Nelson and Winter [8], referred to "working examples" that contain some critical aspects of the routine. Given that knowledge embedded in routines is always to some extent not explicit, but tacit and enacted in practice [32, 47], templates define the "details of how the work gets done, in what sequence, and how its various components and subroutines are interconnected" [19]. They can help us codifying and explicitly representing the tacit part of knowledge, thereby providing an effective way to overcome stickiness to the original context of knowledge [21]. Meanwhile, by codifying and explicitly representing knowledge inherent in routines, templates can also be helpful to the replicatee units in discerning and adopting the so-called "best practices" [55]. This requires to look at the relationship between the sending and receiving units $[24,56]$, as the ability and willingness of replicatees to accept knowledge transferred from the sending unit are also prerequisites for the replicating process of routines [57].

2.2. Routine Networks and the NK Fitness Landscape. Organizations can be considered as certain specific aggregations of "bundle of routines" $[8,48,58,59]$, which can form and change through a process of "combinatorics" [12]. However, it is only until recently that scholars began to focus on how routines affect one another in a more complexityfriendly perspective $[11,36,60]$. For instance, Peng et al. [36] emphasized that looking at routine interrelations is key to understand how routines bundled together to generate organizational capabilities - including both incremental and radical innovation. According to Kremser and Schreyögg [39], this interrelatedness can be attributed to the division of labor in that, due to economics of specialization, organizations while dividing work into "multiple, separate routines" are also required to "securing a sufficient integration of efforts" [39]. This suggests the need for looking not only at the division of multiple specialized routines from the complex organizational task, but also at the interrelationship between these routines. This implies that only by specific routine integration organizations can fully accomplish their organizational task.

Here, research suggests a holistic perspective as organizational routines are embedded in systems of routines $[14,36,61]$. This means that they are systemically integrated and reinforce each other so giving rise to a collective effect, which is greater than only the single contribution of a simple set of individual components [62]. In this line, Argote and Ingram [25] considered the "network of routines" as an important form of organizational memories and the repository of organizational knowledge. Salvato [63] argued that 'the interplay of existing routines', together with individuals' everyday actions, should be treated as real "unit of analysis" rather than single factors. More recently, Sele and Grand [60] investigated the role of both human and nonhuman actants in the connection between routines and considered the interaction of routines as a source of generativity for the whole organizational system.

In this paper, we tried to incorporate some aspects of these networks of routines by using Kauffman's [43] NK model to formalize interactions among these multiple organizational routines. Being originally developed to analyze biological organizations' evolutionary problems [64], Kauffman's NK modeling and fitness landscape theory was then introduced into organizational studies by Levinthal [65]. It eventually became popular in studies on technological innovation [66], strategic planning [67, 68], and organizational learning [69]. NK modeling now can be considered as one of the crucial toolkits for coping with a series of classic organizational problems of search in multidimensional spaces. According to Ganco and Hoetker [68], NK modeling provides a "relatively simple and replicable methodology" addressing organizational problems that cannot be comprehensively understood empirically.

\section{The Model}

3.1. A fitness Landscape Approach to Routine Networks. Based on Nelson and Winter's [8] notion of routines as the "genes" of organizations, Ventresca and Kaghan [70] and Yi et al. [71] suggested to apply the NK model to the study of organizational routines. Through the NK-based fitness landscape, we can build up a description of the linkage between the network of routines and the organizational fit with its environment. For example, let us assume that there are $N$ routines involved in a unit, $R=\left\{r_{1}, r_{2}, \ldots, r_{N}\right\}$, and for each routine $r_{i} \in R(i=$ $1,2, \ldots, N)$, each owning a number of different states - or in other words, different kinds of approaches for unit members to accomplish the corresponding organizational subtasks $S_{i}=$ $\left\{s_{1}, s_{2}, \ldots, s_{A}\right\}$. Thus, according to Kauffman's [43] theory, this routine's contribution to the overall unit fitness fit $_{i}$ would depend not only on its own state, but also on the states of some 


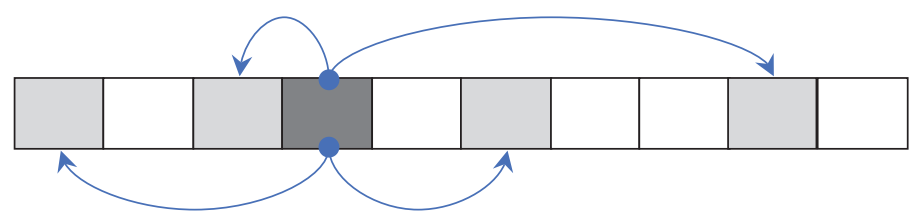

FIGURE 1: Randomly assigned relations between routines $(\mathrm{N}=10, \mathrm{~K}=4)$.
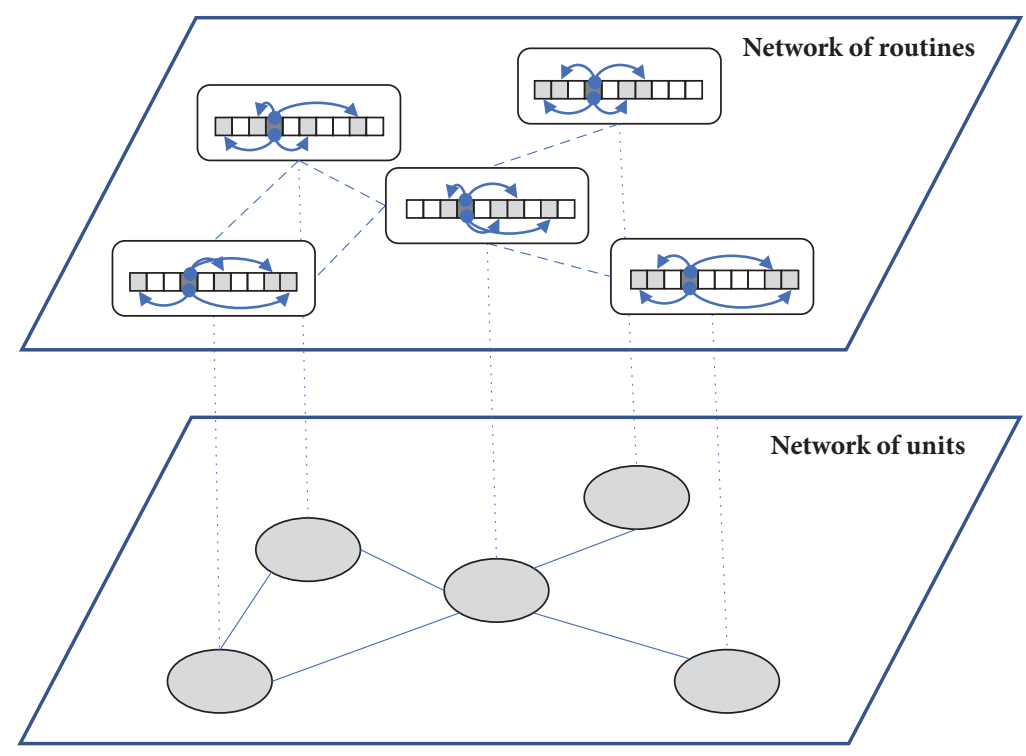

FIGURE 2: Network of units with different routine networks.

other $K$ routines $R_{K}^{(i)}=\left\{r_{1}^{(i)}, r_{2}^{(i)}, \ldots, r_{K}^{(i)}\right\}$ (where $R_{K}^{(i)} \subset R$ and $\left.r_{i} \notin R_{K}^{(i)}\right)$ with which it is interconnected.

Kauffman [43] described two main methods for assigning relations between dependent parts - namely nearest neighbor influences and randomly assigned influences. He then proved that the distribution of $K$ did not dramatically affect the NK model outcomes. In this paper, for simplicity, we adopted the latter strategy and randomly assigned relations between routines [72], as shown in Figure 1.

Thus, we had the following fitness contribution of each routine $r_{i}$,

$$
\begin{aligned}
\text { fit }_{i}=f\left(r_{i} ; r_{1}, r_{2}, \ldots, r_{K} \mid r_{1}, r_{2}, \ldots, r_{K}\right. & \left.\in R_{K}^{(i)}\right), \\
& \quad i=1,2, \ldots, N
\end{aligned}
$$

Here, the value of the key parameter $K$ can be from 0 to $N$ 1. On the one hand, $K=0$ represented a situation in which each of the $N$ routines involved in the unit was independent. On the other hand, with the increase of the value of $K(K>0)$, the change of one routine would influence the performance of other interrelated routines in that any search for improving the configuration of all of them would be more and more difficult [71]. Finally, $K=N-1$ represented another very specific situation in which all routines were interconnected with each other.

The fitness value of the unit with its task environment can be calculated as

$$
\begin{aligned}
& \text { Fitness }=\frac{1}{N} \sum_{i=1}^{N}\left(f_{i}\right) \\
& =\frac{1}{N} \sum_{i=1}^{N} f\left(r_{i} ; r_{1}, r_{2}, \ldots, r_{K} \mid r_{1}, r_{2}, \ldots, r_{K} \in R_{K}^{(i)}\right)
\end{aligned}
$$

according to which the higher the fitness value Fitness, the more adaptive the unit to its living environment.

3.2. Agent Design. In our model, we focused on network characteristics of distributed branches/units within an organization. For the sake of simplicity, we assumed that there was a decentralized organization [73] with unit_num $=40$ units. All these units $G=\left\{g_{1}, g_{2}, \ldots, g_{M}\right\}$ were interdependent entities with similar characteristics, facing a recurring but common problem. They were randomly located in a $100 \times$ 100 lattice and interconnecting with each other with a given probability random_ $p(0 \leq$ random_ $p \leq 1)$. The units constituting the whole organization faced the same environment defined by a landscape described in formulas (1) and (2). We assumed that every unit $g_{j} \in G(j=1,2, \ldots$, unit_num $)$ was considered a heterogeneous agent dealing with a series of organizational subtasks $O T=\left\{o t_{1}, o t_{2}, \ldots, o t_{N}\right\}$ and having a correspondingly specific routine $r_{i} \in R=\left\{r_{1}, r_{2}, \ldots, r_{N}\right\}$ for each task ot $_{i} \in O T(i=1,2, \ldots, N)$ [71]. All routines were interrelated with each other, resulting in interrelationships both within and between bundles of routines, as shown in Figure 2. 
TABle 1: Definition of agent attributes.

\begin{tabular}{|c|c|}
\hline Attributes & Definition \\
\hline unitID & A unique ID number for agent identification. \\
\hline unitNeighbors & $\begin{array}{l}\text { Every unit agent owned a list of neighbors defined by the intraorganizational network } \\
\text { structure. Here, we assumed a classic random network following Erdös and Rényi [74], where } \\
\text { nodes represented unit agents and linkages between nodes - which represented their } \\
\text { neighborhoods - were randomly assigned by the probability value random_ }(0 \leq \text { random } p \leq \\
\text { 1). Given the bounded rationality characterizing organizational actors [75], we assumed that } \\
\text { each unit agent could only interact with and replicate routines from nearest neighbors. }\end{array}$ \\
\hline networkOfRoutines & $\begin{array}{l}\text { Each unit agent had a bundle of routines (i.e., a certain specific vector with " } 0 \text { " or "1" values) to } \\
\text { cope with its series of organizational subtasks. However, rather than being only a simple } \\
\text { collection, these routines were interrelated with each other }[11,39] \text {, so giving rise to a network } \\
\text { of routines. We assumed that this network was randomly generated }[43,72] \text {, as illustrated in } \\
\text { Figure } 1 .\end{array}$ \\
\hline Fitness & $\begin{array}{l}\text { The value of this parameter represented the adaptiveness of a unit agent to its living } \\
\text { environment. In detail, we considered each routine's fitness contribution function } f(\cdot) \text { shown } \\
\text { in formula (1) as a random function mapping the vector }\left(r_{i}, r_{1}, r_{2}, \ldots, r_{K}\right) \text { (where, } \\
\left.r_{1}, r_{2}, \ldots, r_{K} \in R_{K}^{(i)} \text { and } R_{K}^{(i)} \subset R, r_{i} \notin R_{K}^{(i)}, i=1,2, \ldots, N\right) \text { with uniformly distributed values } f i t_{i} \\
\left(0 \leq f i t_{i} \leq 1\right) \text {. Then, we assumed that the fitness value of the unit agent was numerically equal to } \\
\text { the average of the fit value of all the routines involved (as shown in formula (2)). }\end{array}$ \\
\hline
\end{tabular}

Furthermore, we assumed that each routine could generate only two different patterns of action, " 0 " and " 1 ", respectively. Although a specific routine may have more than two states, a set of routines could also be interpreted "as subroutines that constitute a routine of more than two states" [71].

We assumed that each unit agent had attributes as shown in Table 1.

However, as we mentioned in Section 2.1, unit agents often used templates when replicating routines from neighbors. We assumed that a template could not be the exact copy of the network of routines of the replicator unit agent. Indeed, given that at least a part of knowledge embedded in routines is to some extent tacit and enacted in practice $[32,47]$, these differences mimicked in difficulties for replicatee units to explicitly encode routines into templates.

More specifically, we assumed that when replicating activities occurred, the replicatee unit agents obtained a template which was duplicated from the network of routines of the replicator unit agents but with some given probability of errors error $p$ ( $0 \leq$ error $\left._{-} p \leq 1\right)$. This allowed us to mimic errors that may occur during codifying and explicitly representing process of the original set of organizational routines $[18,25]$. However, note that in the model we considered the value of error_ $p$ as constant throughout all simulations. Considering reasons and/or even mechanisms for this kind of errors was out of the scope of our work.

Finally, we considered the environment as a regime of possibilities and constraints for unit agents while accomplishing their organizational task [22]. This environment determined both the organizational subtask series and the fitness landscape as shown in formulas (1) and (2). In order to simulate environmental dynamics, in our model, we adopted a Boolean variable is_env_change? to represent whether the fitness landscape changed or not. On the one hand, when is_env_change? = TRUE, the fitness landscape would randomly vary once after every certain number of simulation rounds $e n v_{c}$ change_P so as to mimic environmental changes occurred in practice. Further, for any env_change_ $P \geq 1$, we have that the lower the value of this variable was, the more rapid environmental changes happened. On the other hand, when is_env_change? = FALSE, it meant that there was no change of the organizational environment. Thus, any values of the variable $e n v_{-}$change_P are meaningless in this situation.

3.3. Rules of the Replicating Activities. According to Hodgson [50], replicating routines from peers is not easy often also due to organizational inertia (e.g., [71, 76]) and bounded rationality [75]. To mimic this, we assumed that unit agents were prone to replicate "superior practices" from neighbors only when they felt competitive pressures, i.e., they had a significantly lower fitness value than the average of neighbors.

Next, a template was generated through explicitly codifying the knowledge embedded in the network of routines of the replicator unit agent $[19,21]$. In this model, we used a vector of " 0 " or " 1 " values as follows:

$$
T P L=\left(t p l_{1}, t p l_{2}, \ldots, t p l_{N}\right)
$$

being templates of a copy of the original series of routines, but allowing each of the element values of the vector $t p l_{k}(0 \leq$ $k \leq N)$ to vary with a certain probability of errors error $-p(0$ $\leq$ error_p $\leq 1)$. Namely,

$$
t p l_{k}= \begin{cases}\left|1-r_{k}\right|, & \text { with a probability error }-p \\ r_{k}, & \text { with a probability }\left(1-\text { error }_{-} p\right)\end{cases}
$$

Here, the value of the coefficient $r_{k}$ was either " 0 " or " 1 ", which represented the two different states (i.e., patterns of actions) of the $k$ th original routine of the replicator unit agent, and $r_{k} \in R=\left\{r_{1}, r_{2}, \ldots, r_{N}\right\}$.

Furthermore, given that replicatee unit agents were always constrained by a specific level of absorptive 


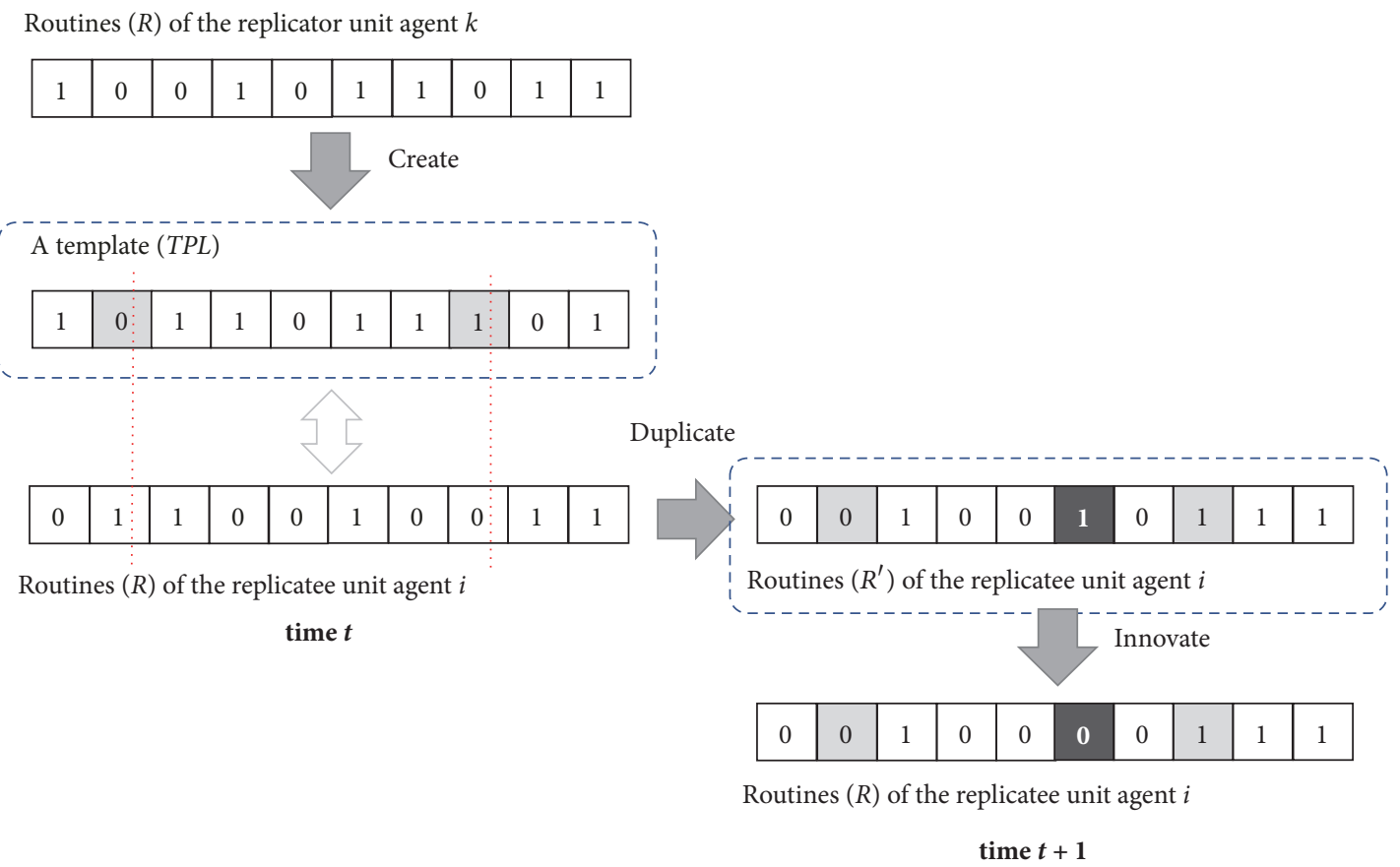

FIgURE 3: Mechanisms of replicating routines between unit agents (unit_AC=2).

capacity $[77,78]$, we assumed that they could not adopt the whole template but only a certain part. Here, we used a variable unit_AC to represent the absorptive capacity of unit agents. Its value numerically equaled the number of routines that replicatee unit agents could change at a time according to the template, unit_AC $=\{1,2,3\}$. Obviously, the higher the value of unit_AC, the higher level of the unit agent's absorptive capacity. In this respect, note that exploring details of agents' absorptive capacities was beyond the scope of our study. For the sake of simplicity, we assumed that the value of unit_AC was assigned at the beginning of the simulation and remained fixed throughout our simulations.

The replicatee unit agents could randomly select a number of routines (which numerically equaled the unit_AC value), which owned different states with those from the template - if they existed, of course. Then, the replicatee unit agents changed the relating routines' states (values) according to the template and generated a new list of routines $R$ '.

Finally, when the new collection of routines $R$ ' was formed, replicatee unit agents checked if they could perform any innovation to match their work of routines (obviously, here, referring to the list of routines $R^{\prime}$ ) and the specific context in which they were living. This was to mimic the "contextdependent" characteristic of organizational routines [22] as well as the interconnection between routines [15, 18, 20,39]. For example, replicatee units could revise parts of routines while considering both the embeddedness of the system of routines [11] and certain fitness landscapes - namely, the units' living environment $[22,42]$. By doing these innovative activities, replicatee unit agents transformed the temporary list of routines $R$ ' into their final network of routines $R$ with newly updated status.

In our model, we assumed that replicatee unit agents could innovate and randomly vary the state of one of their routines with a given probability innovate_p $(0 \leq$ innovate_p $\leq 1)$ - when this was helpful to improve their fitness values.

Figure 3 provides a description of the routine replicating mechanisms between different unit agents. Figure 4 shows a stylized description of the procedure in which unit agents involved in the model should execute during the whole simulation process.

\section{Simulation Experiments, Initialization, and Verification of the Model}

4.1. Simulation Experiments. We constructed our model using NetLogo (https://ccl.northwestern.edu/netlogo/). All the main input settings were shown in Table 2. By applying the unit testing method [79] to each program module and to the whole simulation model, we ensured that our model was comprehensively verified.

The aim of this paper was to investigate the replicating dynamics of routines under the constraints of both the intraorganizational network of unit agents and the routine networks. Furthermore, we considered four other factors, namely, the absorptive capacity of unit agents, the probability of errors occurring during the template-duplicating processes and that of unit agents' innovative activities, and the dynamic characteristic of the organizational environment.

To do so, we first considered one of the simplest cases ("Scenario I"). By assuming that there was no environmental change (in this model, we let the Boolean variable is_env_change $?=$ FALSE, referring to no changes occurred of the fitness landscape) and that there are neither template copying errors nor innovative activities (i.e., error_p $=$ innovate $p=0$ ), we purely investigated the influence of 
TABLE 2: Model input settings.

\begin{tabular}{|c|c|c|}
\hline Input parameters & Default settings & Notes \\
\hline unit_num & $20, \underline{40}, 60,80$ & The number of unit agents involved in the organization \\
\hline random_p & $\{\underline{0.1}, 0.3,0.5,0.7,0.9\}$ & $\begin{array}{l}\text { We adopted a random network model to describe connections between unit } \\
\text { agents. Here, the parameter represented the probability of links between two units }\end{array}$ \\
\hline N_routine & $\{\underline{4}, 6,8,10\}$ & The number of routines involved in the organization \\
\hline A_routine & $\underline{2}$ & The number of states for every routine \\
\hline K_routine & $\left\{\underline{1}, 3, \ldots, N \_\right.$routine -1$\}$ & $\begin{array}{l}\text { The number of routines that affected one certain routine's contribution to the } \\
\text { unit's fitness }\end{array}$ \\
\hline unit_AC & $\{\underline{1}, 2,3\}$ & Absorptive capacity of each unit agent \\
\hline error_p & $\{\underline{0}, 0.1,0.3,0.5,0.7,0.9\}$ & $\begin{array}{l}\text { The probability of errors occurring when replicatee units duplicated routines from } \\
\text { a template }\end{array}$ \\
\hline innovate_p & $\{\underline{0}, 0.2,0.4,0.6,0.8\}$ & The probability of innovative activities for each unit agent \\
\hline is_env_change? & $\{$ FALSE, TRUE $\}$ & A Boolean variable representing whether the environmental changes existed or not \\
\hline env_change_P & $\{200,1000\}$ & The simulation rounds period of environmental changes \\
\hline
\end{tabular}

Note: the underlining values are default settings of the input parameters.

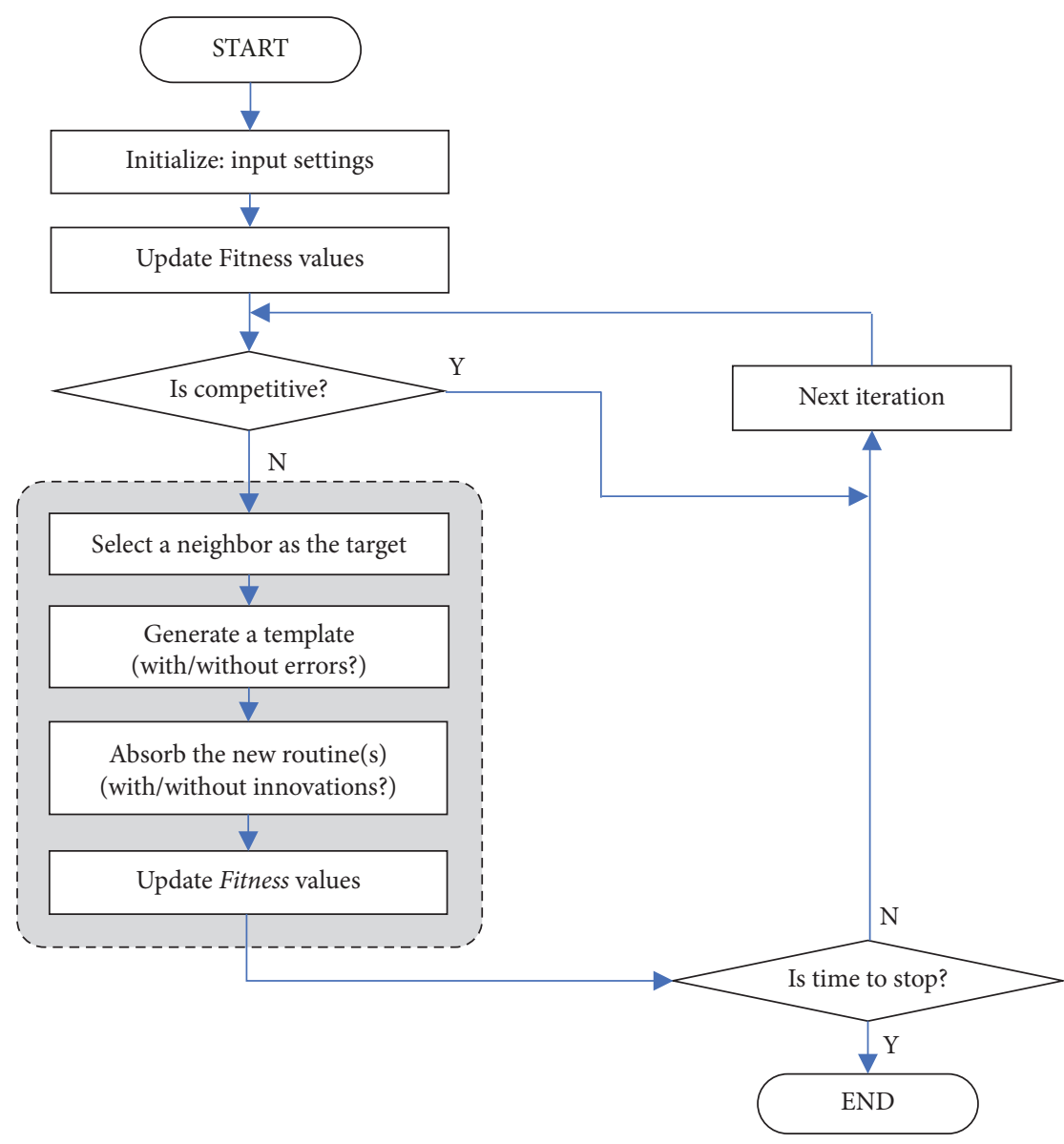

FIGURE 4: Flow chart of replicating activities between unit agents.

both of the two different networks - namely, one is the intraorganizational network of unit agents (determined by the model parameter random_ $p$ ) and the other is the network of routines (represented by the model parameter K_routine) - on routines' replication dynamics.
Then, in "Scenario II", we added variability. On the one hand, we introduced template-duplicating errors (represented by a series of values of the parameter error $p$ ). On the other hand, we assumed that success probability of innovative activities by different replicatee units (represented 
by variations of the parameter values innovate_p) could result in variations of the routine system.

Next, in "Scenario III", we introduced uncertainty of the organizational environment, by varying model parameter values is_env_change? $=$ TRUE, and $e n v_{-}$change_ $P$, so varying the changing rate of the fitness landscape function. This allowed us to examine routine replication within the dynamic organizational environment.

Furthermore, in "Scenario IV", we combined Scenarios II and III to analyze routine replication in a more complex environment, in which internal sources of variability, i.e., template-duplicating errors and innovative activities, were coupled with external sources, i.e., uncertainty of the organizational environment. It is worth noting that these two sources of variations are typical of complex, dynamic environments in the current business landscape, as suggested by Goldstein, et al. [80].

Finally, we developed four aggregate measures to estimate the impact of scenario manipulations. The first one, called steadyTime, measured the number of simulation rounds required by the organization system to arrive at a steady state (e.g., every unit agent found the best bundle of routines that matched its own landscape, and thus there was no replication activity left), if such a specific steady state eventually existed, of course. This is crucial to understand the performance of routine replications as it indicates the replicating speed of routines between different units within the organization. The second one is the average fitness values of all the unit agents. This represented the organizational efficiency that every unit could gain by routine replication, so in principle benefiting the whole organization.

We then also measured the number of replicating and innovative activities occurred among all the unit agents (here, denoted as replicate_num and innovate_num, respectively). When copying routines from their neighbors, unit agents bore some operating cost, i.e., the expenses or resources required to replicating or innovative activities. In general, we hypothesized that the more these activities were performed by unit agents, the higher the operating cost was. In this sense, the number of replicating and innovative activities occurred together could reflect the level of operating cost required by the organization to perform its internal replicating activities.

4.2. Initialization and Verification of the Model. For verifying the correctness of the model, we first considered a very basic scenario in which there were neither variations from template-duplicating errors and innovative activities nor changes in the organizational environment. We let the parameter value unit_num $=20,40,60$, and 80 and keep other variables in the default settings as shown in Table 2. This led to four corresponding but definitely different parameter configurations. In order to determine the ideal number of simulation run for each parameter configuration, we applied the statistical power analysis suggested by Secchi and Seri $[81,82]$. Given the significance level $\alpha=0.01$, the power $1-\beta=$ 0.95 , and the effect size with a small-level $E S=0.1$ according to discussions in [81], we found that 569 runs were sufficient. Then, using the 'smallest effect size of interest' (SESOI) approach, we set simulation runs to 600 times per parameter configuration, which ensured an estimated expected power of 0.96 .

On the other hand, after some pilot runs to study convergence and sensitivity, we decided to let each simulation running for 3000 rounds, each of which representing one routine performance. This allowed us to find a convergence of the routine system to a given steady state, at the same time enabling us to measure one of the most important outputs, i.e., the variable steadyTime.

We then collected output data and calculated the mean values of each variable in each simulation run. Figure 5 shows a summary of simulation results. Figure 5(a) indicates that with the increasing of the number of units involved in the organization, there was a continuous variation of the organization's states, i.e., the values of the three main outputs, steadyTime, replicate_num, and avg_Fitness. It is worth noting here that we did not consider the variable innovate_num as that innovate_ $p=0$ and no innovative activities occurred. First, when connections between units were kept at a very lower level (random_ $P=0.1$ ) and there were only a few units involved (i.e., unit_num $=20$ ), replicating activities occurred randomly but with a very small mean value (see Figure 5(c)). The organization took a short time to reach a steady state (see Figure 5(b)), and the avg_Fitness values followed a very standard normal distribution with mean value approximately equaling 0.5 (see Figure 5(d)). This corroborated our idea of initializing routine networks of all the units randomly.

Furthermore, the increasing number of units obviously increased their interactive opportunities and thus led to the emergence of certain specific patterns of replicating activities. More specifically, the distribution of the variable replicate_num obtained a higher mean value but with a smaller range of variance (see Figure 5(c)). Then, the steadyTime values increased, and the distribution of the variable avg_Fitness followed a partial peak distribution with a bigger mean value (see Figures 5(b) and 5(d)). Again, all these results confirmed the validity of our logics in building and parameterizing the model.

\section{Simulation Results}

Table 2 shows the set of conditions after a series of analyses performed on a testing scenario (as mentioned above) and additional "trial-and-error" experiments. We decided to report our simulation experiments in four subsections corresponding to the four scenarios included in Section 4. To avoid either under- or overpowering our simulation results [81], we let the significance level $\alpha=0.01$, the power $1-\beta=$ 0.95 , while assuming the effect size with a micro level $E S=$ 0.05 due to a pilot study and previous testing experiments. For each of our four scenarios, we followed a full factorial design and calculated the ideal number of simulation runs (denoted as $i_{-}$NofRun) needed for each scenario - as shown in Table 3. Furthermore, following the SESOI approach [81, 82], we set certain running times, see $r_{\_}$NofRun, for each parameter configuration, resulting in an estimated expected power $(E E P)$ of 0.96 . Moreover, we kept each simulation run at 3000 rounds to ensure that the organization system reached a steady state - if this steady state definitely existed. 


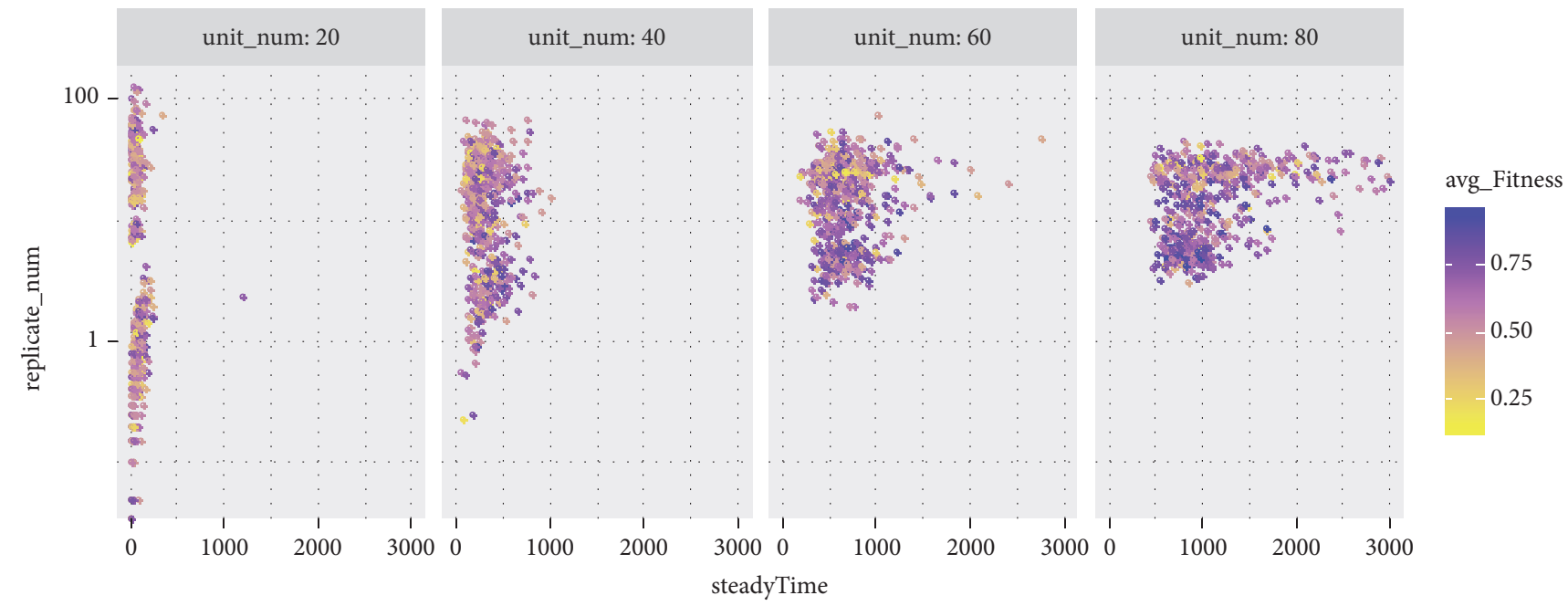

(a)
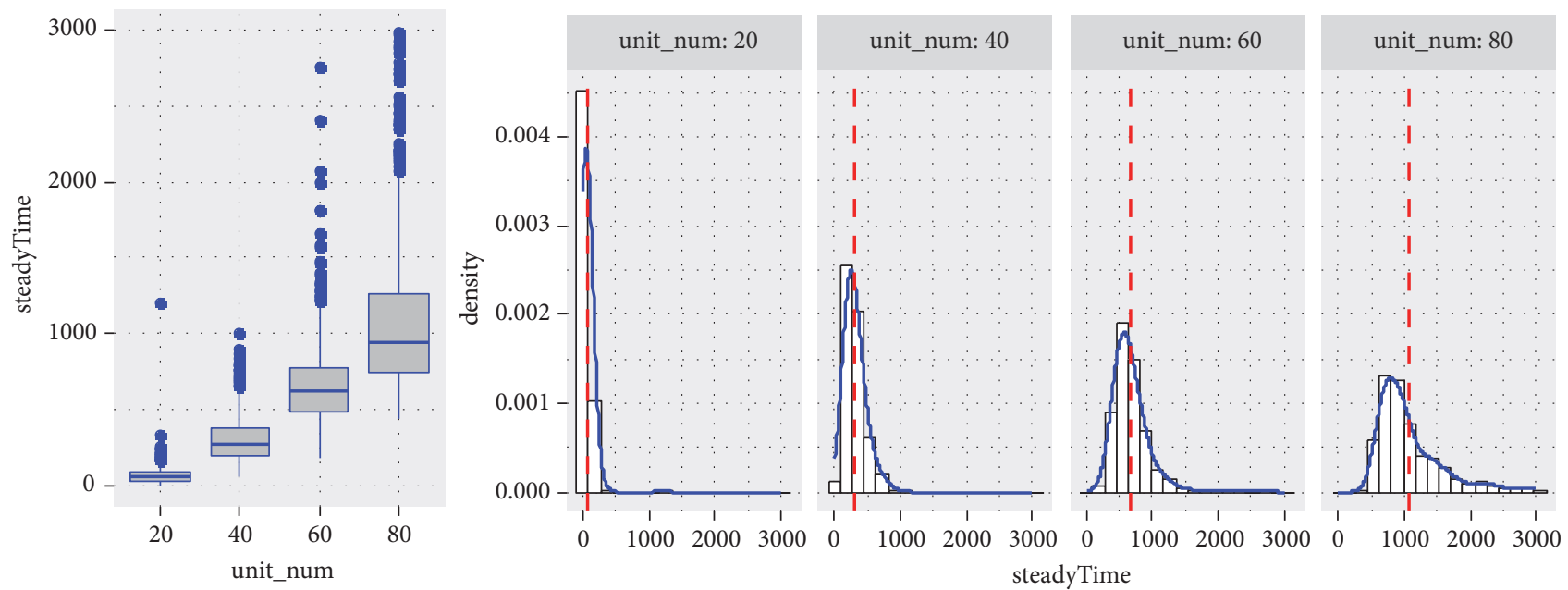

(b)
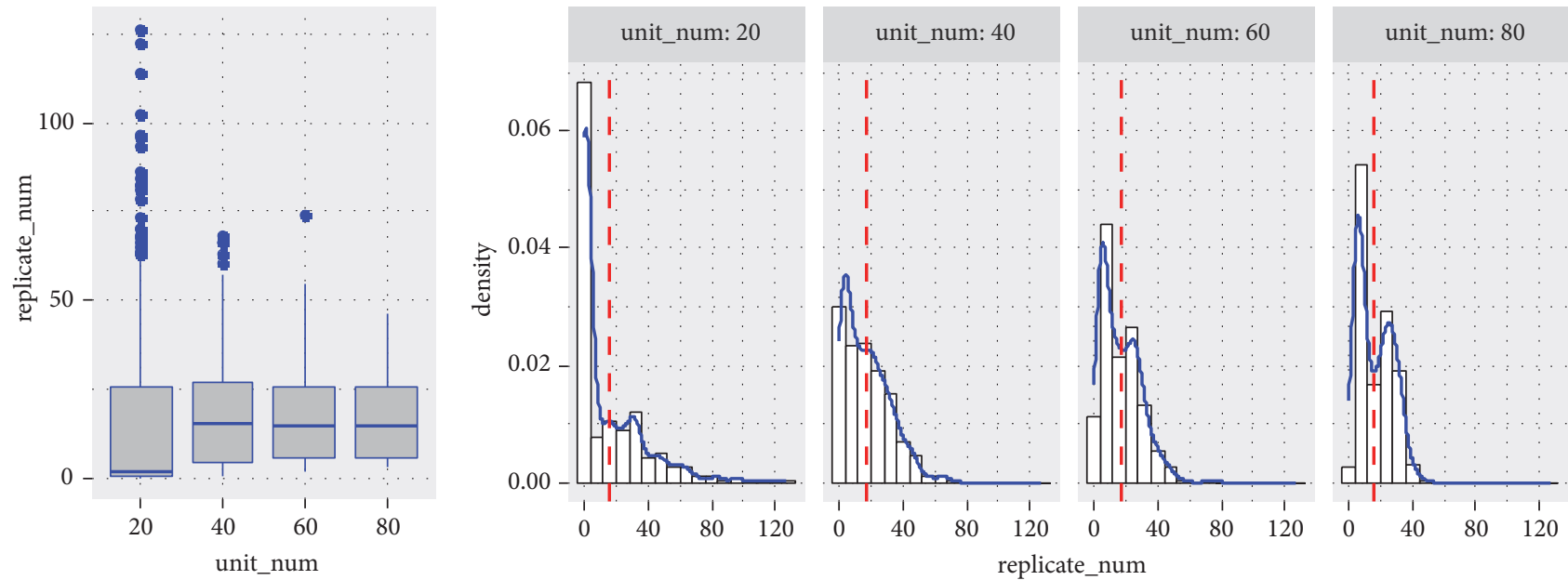

(c)

Figure 5: Continued. 

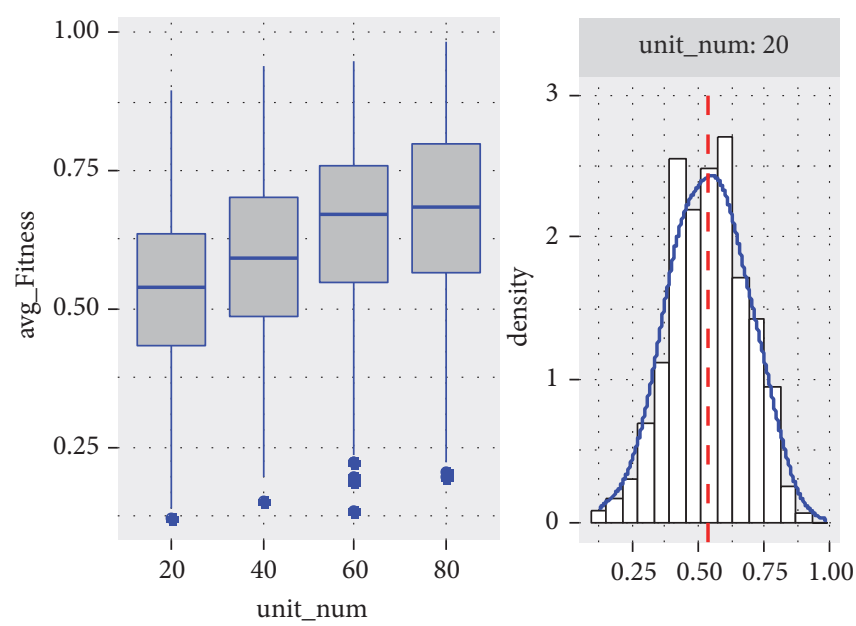
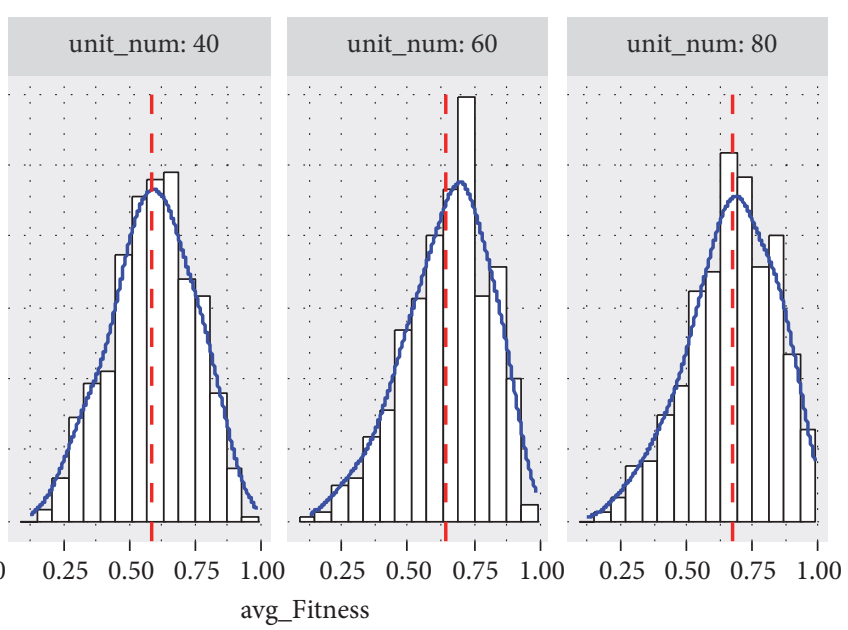

(d)

FIGURE 5: Simulation outputs of the testing scenario: (a) a sketch of the three output variables; (b) distribution of steadyTime; (c) distribution of replicate_num; (d) distribution of avg_Fitness.

(1) Routine Replications within a Static Environment. We assumed unit_num $=40$ and N_routine $=8$ based on the pilot experimental results and assigned random_ $p=0.1,0.3$, 0.5, 0.7 and 0.9, and K_routine $=1,3,5$ and 7, to represent different density levels of both the network of units and that of routines, respectively. We let unit_AC $=1,2$ and 3 to represent different levels of the units' absorptive capacity. For each configuration of these three parameters and with default settings of the other parameters as shown in Table 2 (here, we named this as Scenario I), we collected all the 400 simulation running results and calculated mean values of the three outputs, i.e., steadyTime, replicate_num, and avg_Fitness but excluded the variable innovate_num because we removed any possibility of innovative activities due to the parameter innovate_ $p=0$ in this scenario.

Figure 6 shows simulation results of the other three outputs - namely, steadyTime, replicate_num, and avg_Fitenss. We found that the network density of both units and routines had an impact on routine replication processes, so leading to different performance of the organization. However, their effects were also determined by another important variable, namely, the units' absorptive capacity unit_AC. First, when the parameter unit_AC $=1$, units were limited within a very narrow space when searching target neighbors for replication. With this constraint, even increasing the unit network density (random_p from 0.1 to 0.9 ) could increase the probability for units to link with each other, and all the three outputs steadyTime, replicate_num, and avg_Fitness could increment only minimally. On the other hand, increasing the routine network density (K_routine from 1 to 7 ) had no significant influence on the outputs steadyTime. However, it improved both replicate_num and avg_Fitness with a limited margin except at a very medium value $K_{-}$routine $=3$ when the unit network density random_ $p=0.7$, which resulted in a decrease of the avg_Fitness value (see the left part in Figures 6(a), 6(b), and $6(\mathrm{c})$ ).
Next, when the parameter unit_AC $=2$ and 3, we found that the organization system spent a longer time to arrive at a steady state (i.e., a higher steadyTime value, see the medium and right parts in Figure 6(a)). More replicating activities occurred (i.e., a high replicate_num value, see the medium and right parts in Figure 6(b)), and so a higher level of fitness was reached (i.e., a high avg_Fitness value, see the medium and right parts in Figure 6(c)). In this situation, the lower the value of the variable random_ $p$ was, the greater the effects of its increments to the organizational system were.

On the other hand, although variation of $K \_$routine values had a weak effect when the variable random_ $p$ took low values (e.g., random_ $p=0.1$ and 0.3 when $u n i t_{-} A C=2$; or random_ $p$ $=0.1$ when unit_AC $=3$ ), achieving an optimal fitness when taking a medium value (here, for example, K_routine $=3$ or 5) and the variable random_ $p$ with higher values (i.e., random_ $p=0.5,0.7$ and 0.9 ) was extremely beneficial for the organization. Obviously, in this case, reaching a steady state required more time.

However, if we increased K_routine to a significantly higher value (e.g., in our model, K_routine $=7$ ), the system took less time to reach a steady state, but the increasing values of replicate_num and the decreasing values of avg_Fitness indicate that the organization undertook more replication activities. This permitted to overcome resistance against change due to the strong ties between different routines, yet with no obvious benefits for organization adaptiveness.

To sum up, if unit agents had only very limited absorptive capacity, variations of both the unit and routine networks' density do not improve organizational performance. If we consider the cost for unit agents to manage their relationship with partners, the contribution of the replicating process of organizational routines to the organization performance depends on an optimal configuration of the two types of networks - i.e., the network of units in the organization represented by the variable random_ $p$ and that of routines represented by the variable $K_{-}$routine. 
TABLE 3: Calculating running times needed for each scenario based on power analysis.

\begin{tabular}{|c|c|c|c|c|c|c|c|}
\hline Scenarios & Configura & of paramet & $\mathrm{CoP})$ & & $i \_$NofRun & $r \_$NofRun & EEP \\
\hline \multirow{8}{*}{$\begin{array}{l}\text { Scenario I } \\
(\text { is_env_change? = } \\
\text { FALSE) }\end{array}$} & \multicolumn{4}{|c|}{ Variables and values: } & \multirow{8}{*}{382} & \multirow{8}{*}{400} & \multirow{8}{*}{0.96} \\
\hline & K_routine & \multicolumn{2}{|c|}{ random_p } & unit_AC & & & \\
\hline & 1 & \multicolumn{2}{|c|}{0.1} & 1 & & & \\
\hline & 3 & \multicolumn{2}{|c|}{0.3} & 2 & & & \\
\hline & 5 & \multicolumn{2}{|c|}{0.5} & 3 & & & \\
\hline & 7 & \multicolumn{2}{|c|}{0.7} & & & & \\
\hline & \multicolumn{4}{|c|}{0.9} & & & \\
\hline & \multicolumn{4}{|c|}{$\begin{array}{l}\text { CoP: } \Phi\left(K_{\text {_routine }}\right) \times \Phi\left(\text { random_}_{-}\right) \\
\times \Phi\left(\text { unit_AC }^{(1)}\right. \\
=4 \times 5 \times 3=60\end{array}$} & & & \\
\hline \multirow{9}{*}{$\begin{array}{l}\text { Scenario II } \\
(\text { is_env_change? }= \\
\text { FALSE) }\end{array}$} & \multicolumn{4}{|c|}{ Variables and values: } & \multirow{9}{*}{382} & \multirow{9}{*}{400} & \multirow{9}{*}{0.96} \\
\hline & K_routine & errc & & innovate_p & & & \\
\hline & 1 & 0 & & 0.2 & & & \\
\hline & 3 & 0 & & 0.4 & & & \\
\hline & 5 & 0 & & 0.6 & & & \\
\hline & 7 & 0 & & & & & \\
\hline & \multicolumn{4}{|c|}{0.9} & & & \\
\hline & \multicolumn{4}{|c|}{$\begin{array}{l}\mathrm{CoP}: \Phi\left(K_{\text {_routine }}\right) \times \Phi\left(\text { error_}_{-} p\right) \\
\times \Phi\left(\text { innovate }_{-} p\right)\end{array}$} & & & \\
\hline & \multicolumn{4}{|c|}{$=4 \times 5 \times 3=60$} & & & \\
\hline \multirow{8}{*}{$\begin{array}{l}\text { Scenario III } \\
(\text { is_env_change? }= \\
\text { TRUE) }\end{array}$} & \multicolumn{4}{|c|}{ Variables and values: } & \multirow{8}{*}{253} & \multirow{8}{*}{260} & \multirow{8}{*}{0.96} \\
\hline & K_routine & random_p & unit_AC & env_change_P & & & \\
\hline & 1 & 0.1 & 1 & 200 & & & \\
\hline & 3 & 0.3 & 2 & 1000 & & & \\
\hline & 5 & 0.5 & 3 & & & & \\
\hline & 7 & 0.7 & & & & & \\
\hline & & 0.9 & & & & & \\
\hline & \multicolumn{4}{|c|}{$\begin{array}{l}\text { CoP: } \Phi(\text { K_routine }) \times \Phi(\text { random_p }) \\
\times \Phi(\text { unit_AC }) \times \\
\Phi(\text { env_change_P })=4 \times 5 \times 3 \times 2=120\end{array}$} & & & \\
\hline \multirow{8}{*}{$\begin{array}{l}\text { Scenario IV } \\
(\text { is_env_change? }= \\
\text { TRUE })\end{array}$} & \multicolumn{4}{|c|}{ Variables and values: } & & & \\
\hline & K_routine & error $_{-} p$ & innovate_p & $e n v \_c h a n g e \_P$ & & & \\
\hline & 1 & 0.1 & 0.2 & 200 & & & \\
\hline & 3 & 0.3 & 0.4 & 1000 & & & \\
\hline & 5 & 0.5 & 0.6 & & 253 & 260 & 0.96 \\
\hline & 7 & 0.7 & & & & & \\
\hline & & 0.9 & & & & & \\
\hline & $\begin{array}{l}\operatorname{CoP}: \Phi\left(K_{-}\right. \\
\times \Phi(\text { innov } \\
3 \times 2=120\end{array}$ & $\begin{array}{l}n e) \times \Phi(e r r c \\
) \times \Phi\left(e n v_{-} c\right.\end{array}$ & $\left.e \_P\right)=4 \times 5 \times$ & & & & \\
\hline
\end{tabular}

Notes: (1) here, the function $\Phi(X)$ represented the number of different levels involved in the variable $X$.

(2) Routine Replications with Template-Duplicating Errors and Units' Innovative Activities. In this section, we considered two important factors of variation. One is the probability of errors that may occur when we duplicate routines from the template. The other one is the probability of innovation for unit agents while they replicate routines from their partners. We assumed the values of the parameter error $\_p=0.1,0.3$, $0.5,0.7$, and 0.9 and another one innovate_p $=0.2,0.4$ and 0.6, representing different levels of the two kinds of events' occurrence probabilities, respectively. According to simulation results of Scenario I (as shown in Figure 6), we selected one of the most representative but not extreme cases by letting random_ $p=0.5$ and unit_AC $=2$ and kept the other default settings as Scenario I. Then, we separately investigated the impact of variation from both template-duplicating errors and innovative activities of unit agents. 

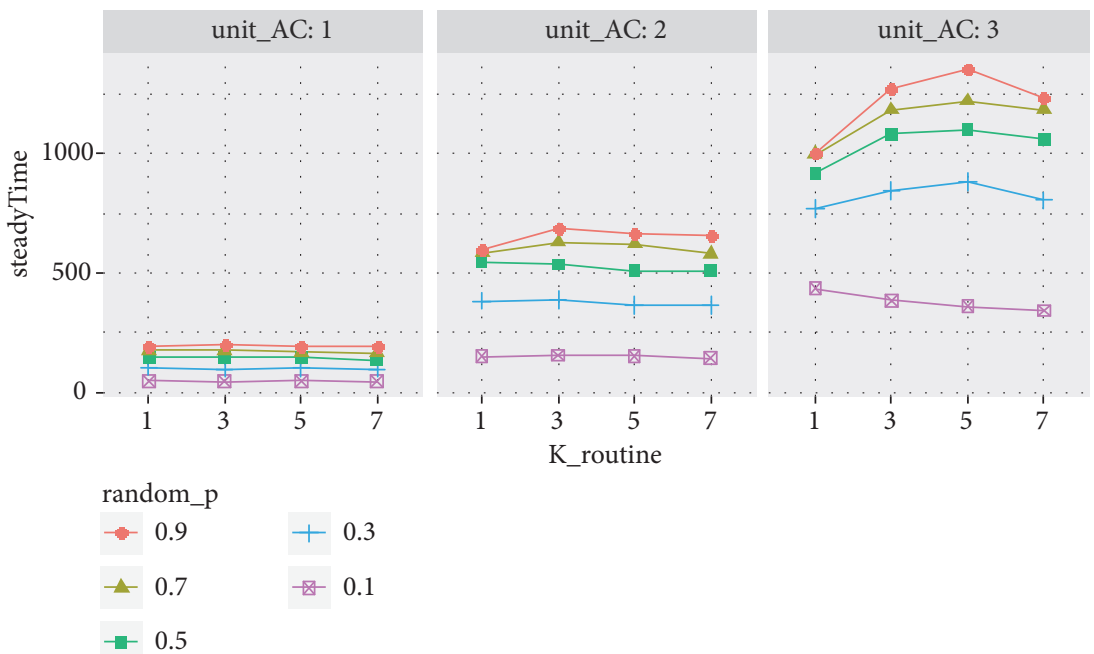

(a)

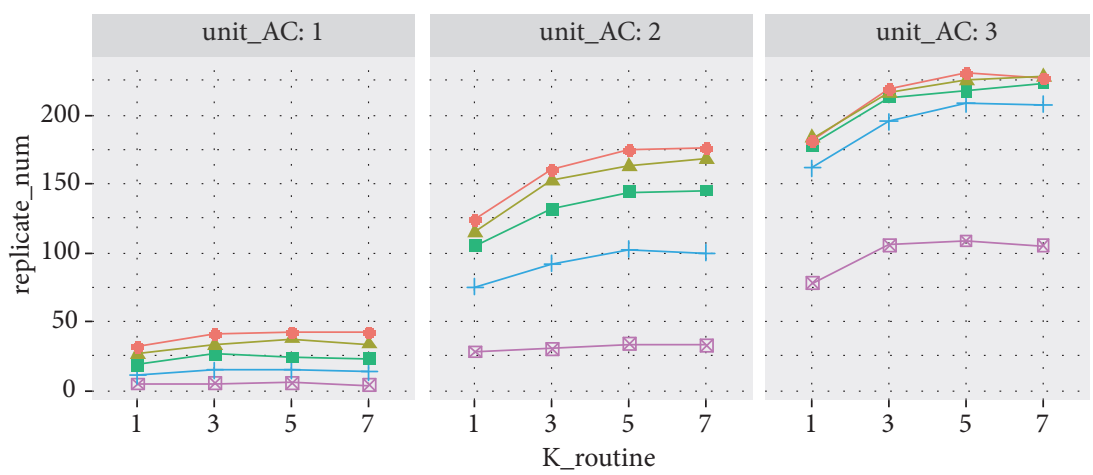

random_p

$$
\begin{array}{ll}
-0.9 & +0.3 \\
-0.7 & \varangle 0.1 \\
-0.5 &
\end{array}
$$

(b)

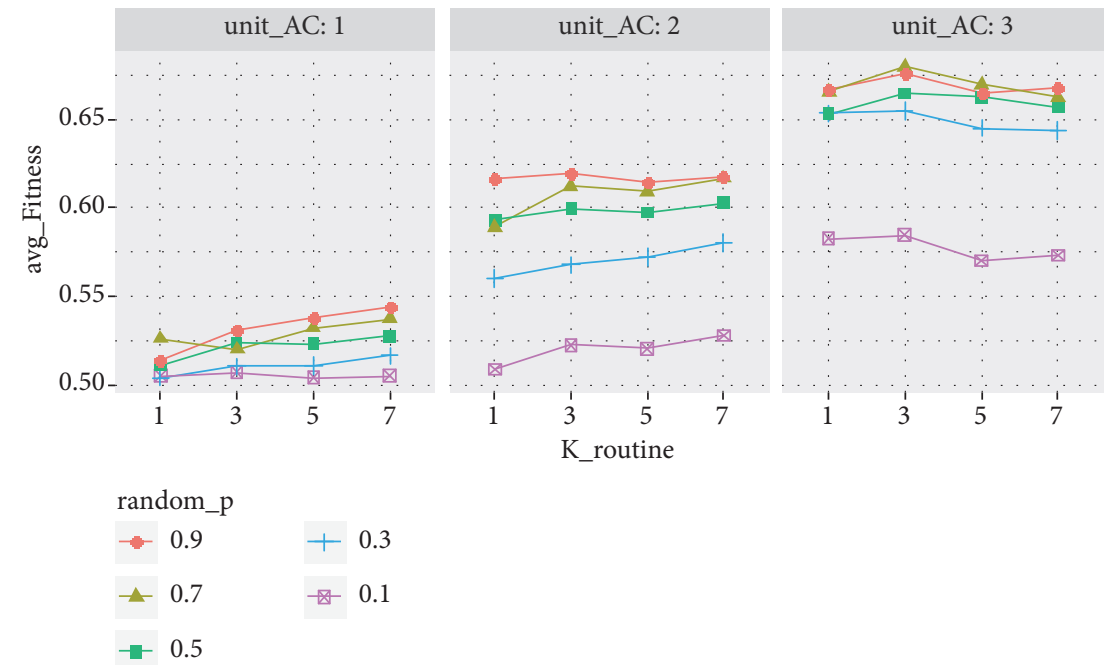

(c)

FIGURE 6: Simulation outputs within a static environment (Scenario I): (a) steadyTime values; (b) replicate_num values; (c) avg_Fitness values. 
Figure 7 shows that these induced variations created opportunities for replicatee units with significant benefit to the fitness of the whole organization. However, this came to the cost of more replicating and innovative activities required and so more time is required by the organizational system to reach a steady state. First, increasing network density of routines (i.e., K_routine) meant a tighter coupling relation between these routines. Therefore, the organizations spent more time and were more involved in replicating and innovative activities (see Figures 7(a), 7(b), and 7(c)). However, we found that when the variable error_p equaled low values (e.g., error_p $=0.1$ and 0.3 ), a medium value of K_routine (i.e., K_routine = 3) still existed which was beneficial to the organization's adaptiveness (see Figure 7(d)).

Second, in case of variation from template-duplicating errors, on the one hand, the higher was the value of the variable error $p$ (e.g., error $p=0.9$ ), the more randomness occurred during the replicating activities, which could result in higher heterogeneity of routine networks between unit agents. On the other hand, very low values of error $p$ (e.g., error_ $p=0.1$ ) caused less fluctuations to the whole routine system. Therefore, these two cases were both beneficial for the organization to economize on the time required to reach a steady state (as shown in Figure 7(a)). Further, increasing innovate_ $p$ values mainly led to more innovative activities among unit agents (see Figure 7(c)). This had a significant influence both on steadyTime and replicate_num only when error $\_$p equaled low values (see Figures $7(\mathrm{a})$ and $7(\mathrm{~b})$ ). It also influenced the avg_Fitness when error_ $p$ did not equal medium values - i.e., error_ $p=0.1$ or 0.9 (see Figure $7(\mathrm{~d})$ ).

Furthermore, variations from both template-duplicating errors and unit innovations led to an increase of replicating and innovative activities at the same time reducing average fitness values. However, when $K_{-}$routine values increased from 1 to 7 , especially when $K_{-}$routine $=7$, the influence of these variations decreased. In these cases, for different error_ $p$ values (from 0.1 to 0.9 ), the three outputs steadyTime, innovate_num, and avg_Fitness tended to converge (see Figures $7(\mathrm{a}), 7(\mathrm{c})$, and $7(\mathrm{~d}))$.

(3) Routine Replications in Dynamic Environments. In this section, we investigated routine replicating processes in a dynamic environment by letting the variables is_env_change? $=T R U E$ and env_change_ $P=200$ and 1000 , respectively. This was to mimic different changing rates of the organizational environment. For the rest, we kept the other input settings as in Scenario I. In this case, given that environmental changes were pervasive, we expected that the organization system as a whole could not arrive at any steady state. Given that the output value steadyTime had no meaning here and that by assuming the variable innovate $p=0$ the output values innovate_num always equaled 0 , we reported here only two simulation outputs including replicate_num and avg_Fitness.

Figure 8 shows that when the fitness landscape changed, we found that the absorptive capacity level of unit agents has a strong effect on routine replicating activities so determining the average fitness value of all the units involved.

First, when absorptive capacity was minimal (e.g., unit_AC $=1$ ), unit agents were very constrained to search for appropriate routine candidates from their neighbors. Independently from the degree of connections between routines (i.e., the K_routine values) or that between unit agents (i.e., the random $p$ values), and the changing rate of the organizational environment (i.e., the different env_change_P values), the average number of unit agents involved in replicating activities (i.e., replicate_num) and the average fitness of all the unit agents (i.e., avg_Fitness) did not have a significant effect. In addition, we also found that environmental changes influenced both these two outputs even more with the connection density between units increased (e.g., increasing the random_ $p$ values from 0.1 to 0.9 ) (see the left part of Figures $8(\mathrm{a})$ and $8(\mathrm{~b})$ ).

Secondly, higher levels of absorptive capacity (e.g., unit_AC $=3$ ) implied that every unit could easily find its ideal routine targets for replication regardless of the pace of environmental changes. To some extent, this explained why variations of env_change_P values had less impact on the replicate_num values (see the right part of Figure 8(a)). Further, although both replicate_num and avg_Fitness were very sensitive to variations of the connection degree between units in case of low values of random_p (e.g., random_ $p=0.1$ ), the variation of random_ $p$ had no effect when unit agents were highly connected with each other (i.e., random_ $p=0.5,0.7$ and 0.9).

Furthermore, when the variable unit_AC equaled a medium value (e.g., unit_AC $=2$ ), we found a correlation between the increasing rapidity of environmental changes (e.g., decreasing env_change_P from 1000 to 200) and less replicating activities (see the middle part of Figure 8(a)). This was especially true when the network density of unit agents and routines was high (e.g., K_routine $=5$ and 7 and random_ $p$ $=0.7$ and 0.9 ).

Finally, Figure 8(b) shows that when unit_AC $=2$ and 3 , the organization's fitness (i.e., the avg_Fitness values) decreased when organizational environment varied more rapidly (e.g., decreasing env_change_P from 1000 to 200) (see the middle and right parts of Figure 8(b)). In addition, we found a positive relationship between K_routine and avg_Fitness, except when the unit network density was low (e.g., random_p $=0.1$ ). In this cases, there was an optimal avg_Fitness when K_routine had a medium value (i.e., K_routine $=3$ ), similarly to what occurred in a static environment.

(4) Routine Replications with Internally and Externally Coupled Uncertainties. In this section, we combined Scenarios II and III to examine the influence of coupled internal variations and external uncertainties. To do this, we set up random_ $p=$ 0.5 ; unit_AC $=2$; error_$_{-} p=0.1,0.3,0.5,0.7$, and 0.9 ; innovate $-p$ $=0.2,0.4$ and 0.6 ; is_env_change? $=$ TRUE; and env_change_P $=200$ and 1000, while the other input parameters were the same default settings of scenarios I, II, and III.

Figure 9 shows that within a dynamic environment, more replicating and innovative activities were required to cope with more coupling of routines (i.e., routines' connection density parameter K_routine). Moreover, we found that although more template-duplicating errors (i.e., the variable error_p) had less impact on unit agents' innovative activities, 


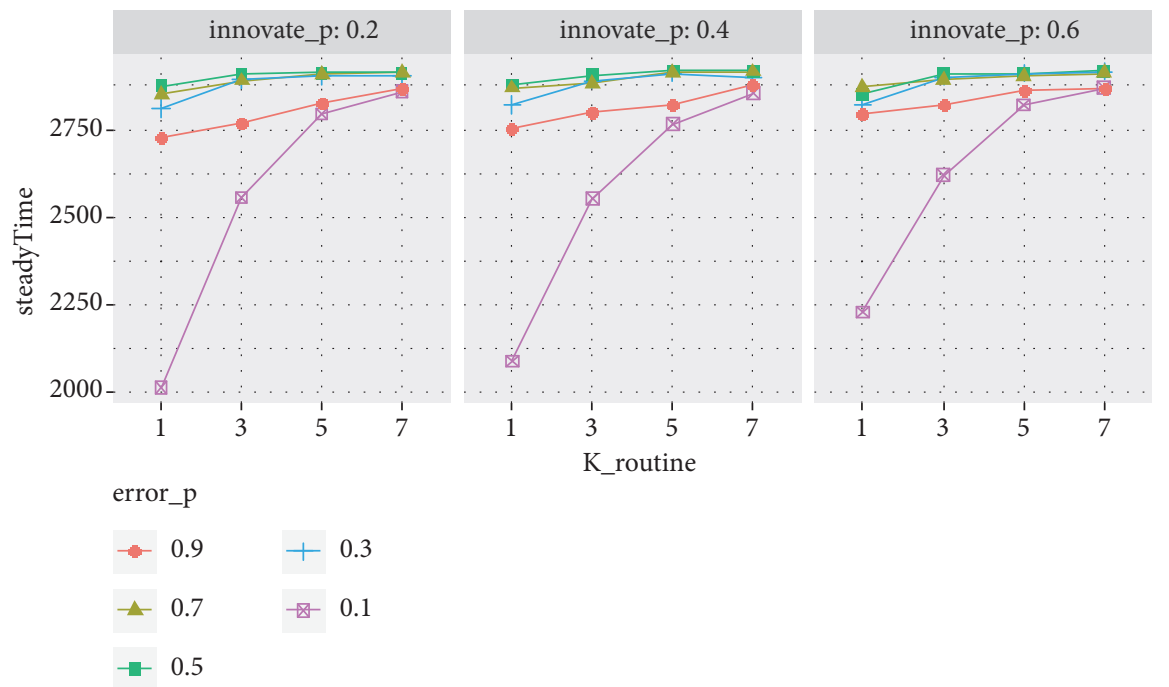

(a)

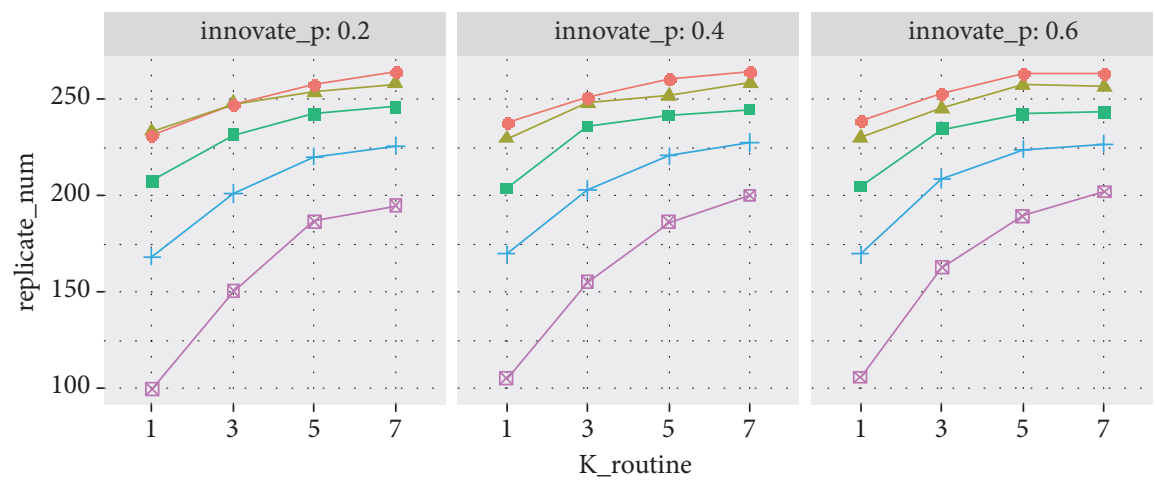

error_p
$-0.9+0.3$
$-0.7 \quad-0.1$
-0.00
$+0.5$

(b)

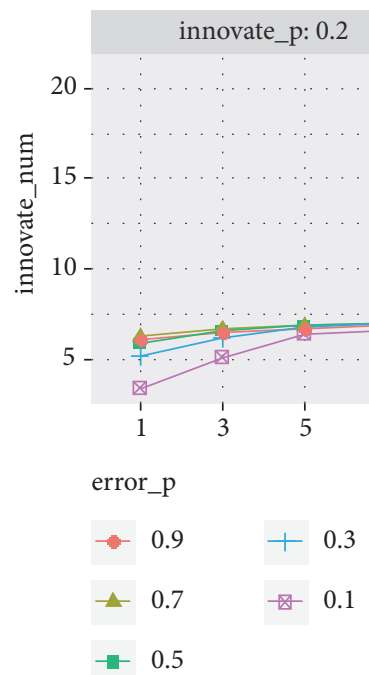

innovate_p: 0.4 innovate_p: 0.6

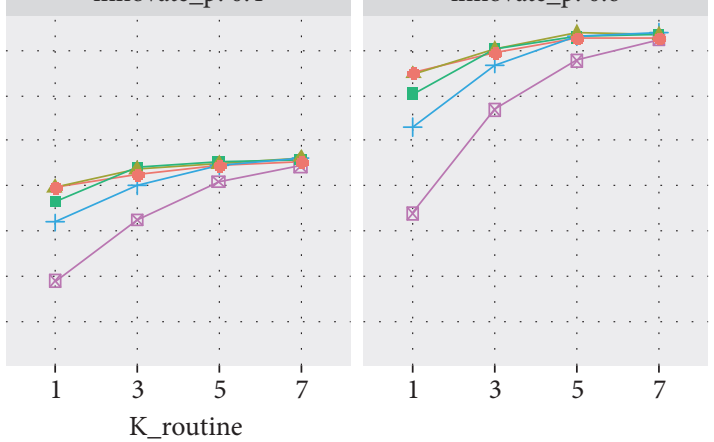

K_routine

(c)

FIgURE 7: Continued. 


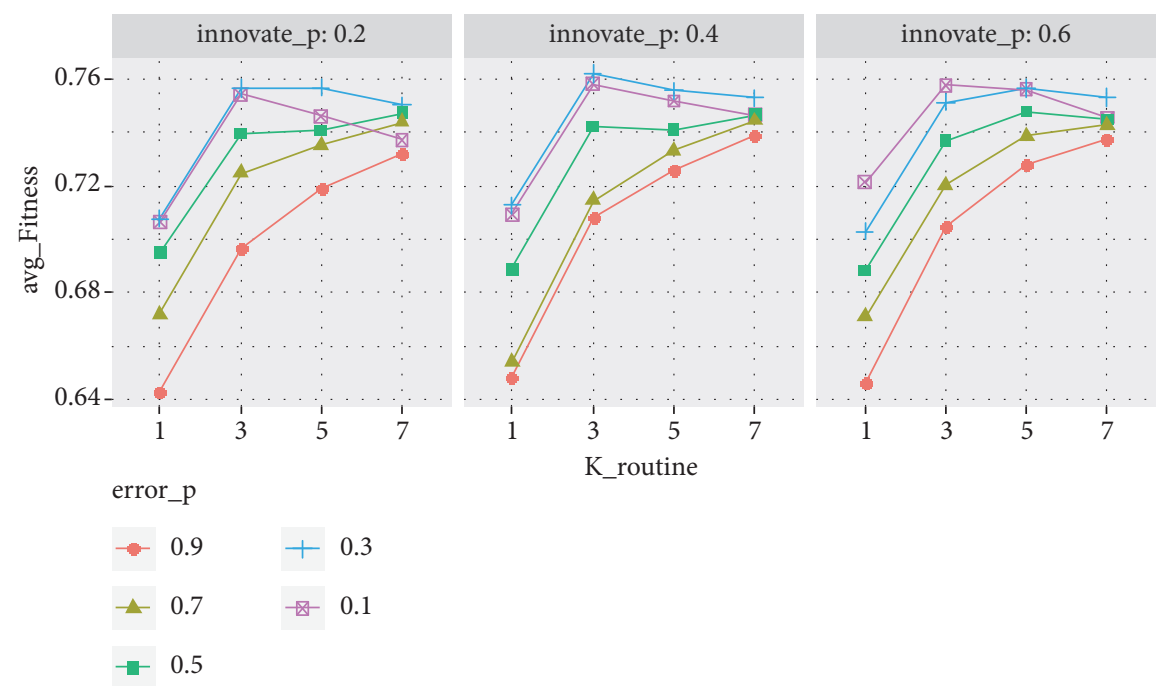

(d)

FIGURE 7: Simulation results with template-duplicating errors and units' innovative activities (Scenario II: random_p $=0.5$ and unit_AC $=2$ ): (a) steadyTime values; (b) replicate_num values; (c) innovate_num values; (d) avg_Fitness values.

this caused more randomness to the routines system, and thus required more routine replicating activities. On the other hand, while increasing innovate_ $p$ values could only promote innovative activities of unit agents, this determined less influence of the number of unit agents involving in routine replicating activities (see Figures 9(a) and 9(b)). This was especially true when errors in template-duplication were low (e.g., error $\_p=0.1$ and 0.3 ).

Secondly, when the organizational environment changed rapidly, density variations in unit agents and routine networks did not have any significant influence on the level of innovative activities, except when less template-duplicating errors (e.g., error_ $p=0.1$ ) were matched with a slowly changing environment (e.g., env_change_P $=1000)$. In this situation, any increase of routine coupling (e.g., K_routine values varied from 1 to 7) led to more innovative activities. However, differences of both replicating and innovative activities caused by variation of either $K_{-}$routine or error_p values decreased with the increasing rapidity of environmental changes.

Figure 9(c) shows that environmental changes had a negative effect on organization adaptiveness. Furthermore, in case internal and external variations were not particularly prominent (i.e., error $\_p=0.1$ and 0.3 , and env_change_ $P=$ $1000)$, medium values of K_routine (e.g., K_routine $=3$ ) were still beneficial to the optimal fitness of the organization. Otherwise, we found a positive relation between K_routine and avg_Fitness values when $K_{-}$routine $=1$, but this relation was not significant when $K \_$routine value was high (e.g., K_routine $=5$ ).

Furthermore, in a slowly changing environment (e.g., env_change_ $P=1000)$, with the increase of templateduplicating errors (i.e., increasing error $p$ from 0.1 to 0.9 ), the average fitness values decreased. However, this negative relation decreased when the speed of environmental changes was higher (see the bottom part of Figure 9(c)).
On the other hand, no matter whether the organizational environment changed slowly or rapidly, higher values of innovate_p only resulted in more innovative activities (i.e., the increase of innovate_num values) as a means to cope with the randomness caused by both internal variations and external dynamics, and this did not have significant effects on the output avg_Fitness (see the medium and right parts of Figure 9(c)).

Finally, by comparing the bottom and upper parts of Figures 9(a) and 9(c), we found when the organizational environment was moderate (e.g., env_change_ $P=1000$ ), the routine system could reach a higher level of fitness (i.e., the avg_Fitness values) but with less replicating activities in contrast to that within a dramatically changing environment (e.g., env_change_P $=200$ ). These findings are consistent with Rivkin's arguments in [51].

\section{Discussions and Conclusions}

In this paper, we considered replication of organizational routines as some specific "template-based" activities between different units within an organization $[19,21,30]$ and developed an agent-based model to simulate the routine replicating dynamics from the "bottom-up" approach [83]. We considered two distinguished types of network contexts, the first one referred to connections between (geographically) distributed units [24] and the other one was the coupling relation between different routines $[11,36,60,63]$. By depicting the former as a random network model [74] and the latter as a NK-based fitness landscape [43], we constructed an agent-based model that allowed us to zoom in the routine replicating process in detail. At the same time, the model permitted to consider the impact of structural factors, such as the two different types of network contexts, the internal variations from both template-duplicating errors 


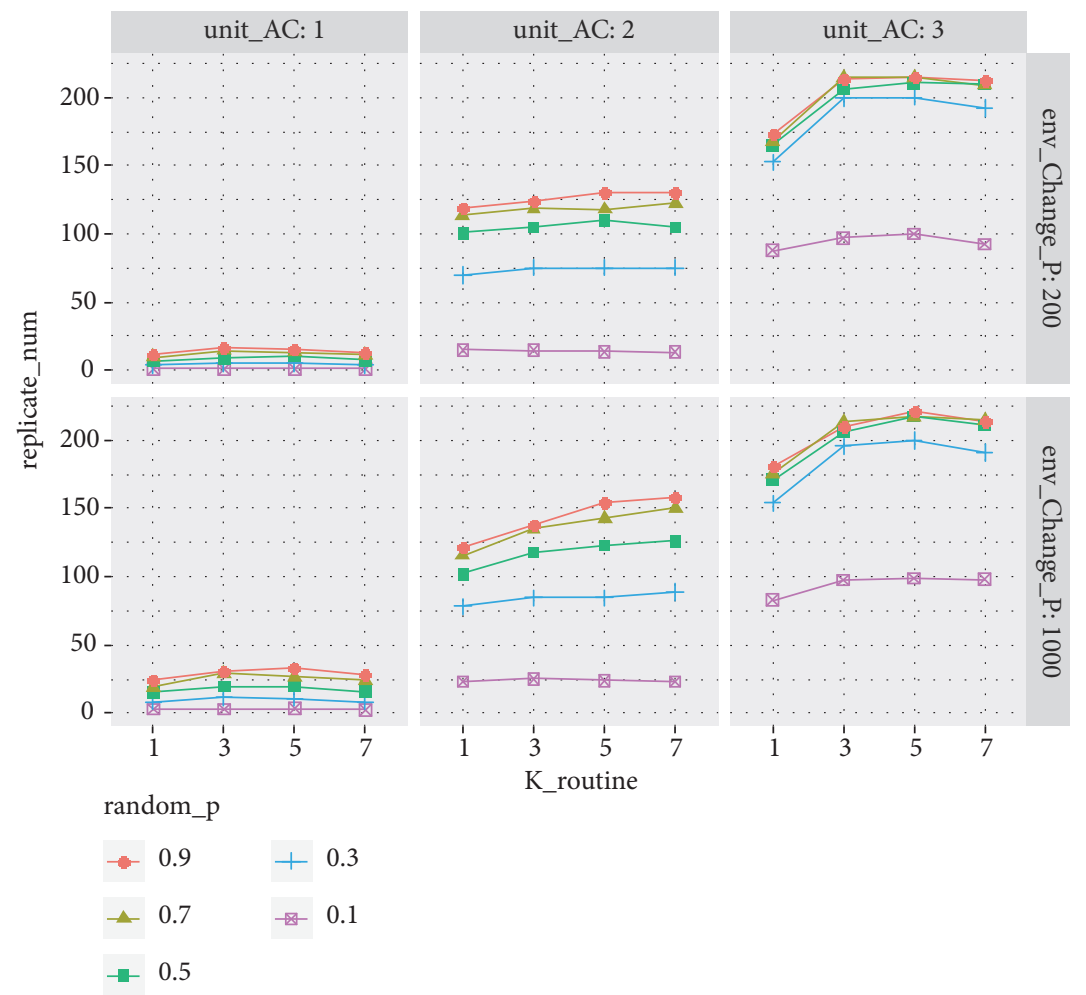

(a)

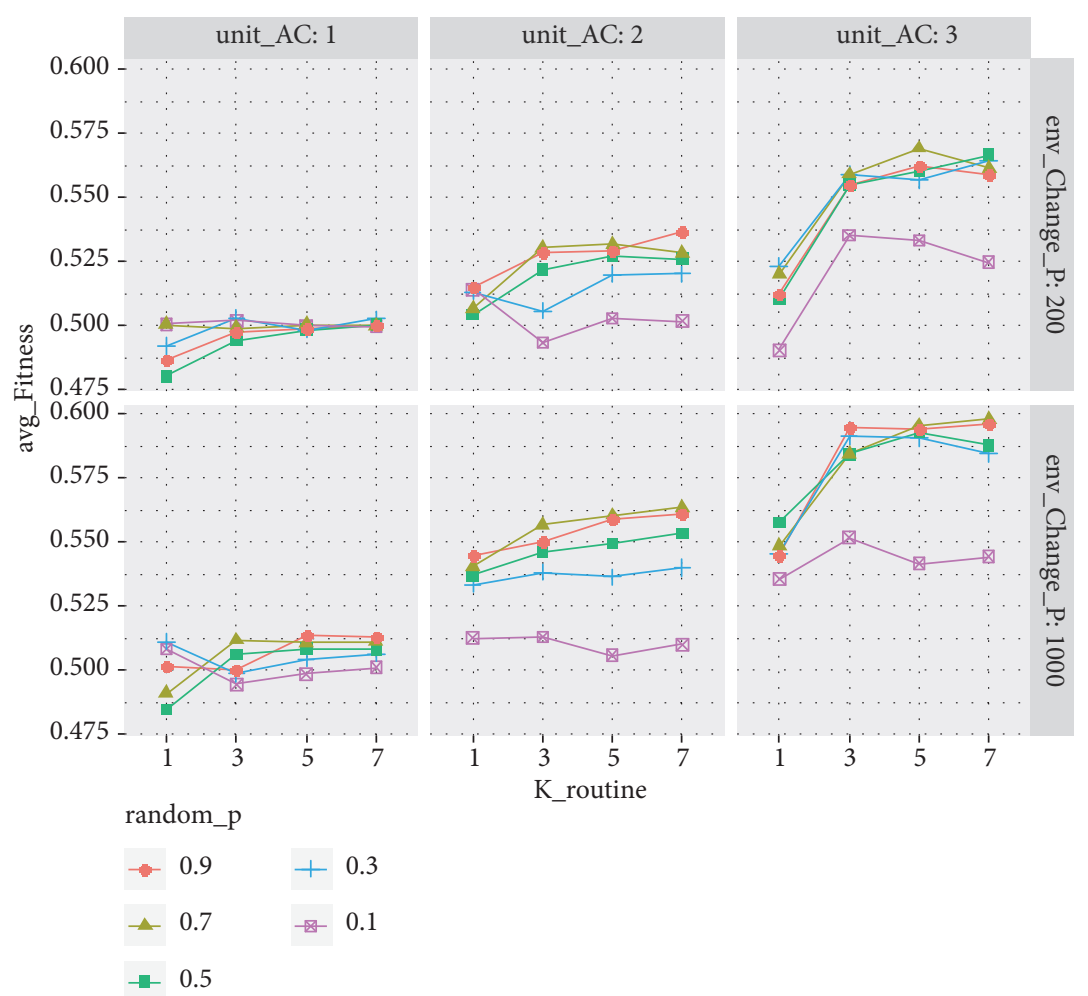

(b)

FigURE 8: Simulation results in dynamic environments (Scenario III): (a) replicate_num values; (b) avg_Fitness values. 


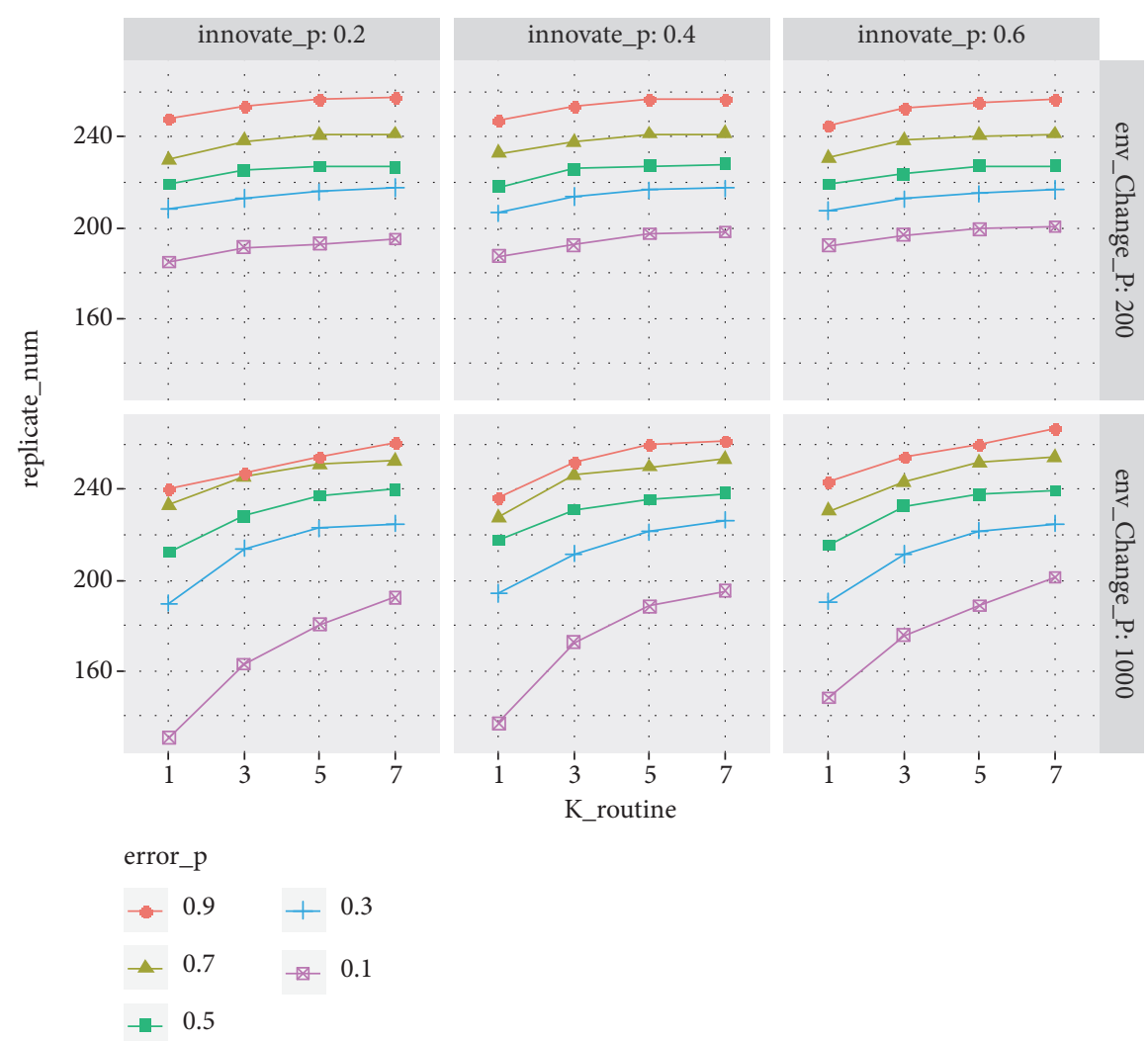

(a)
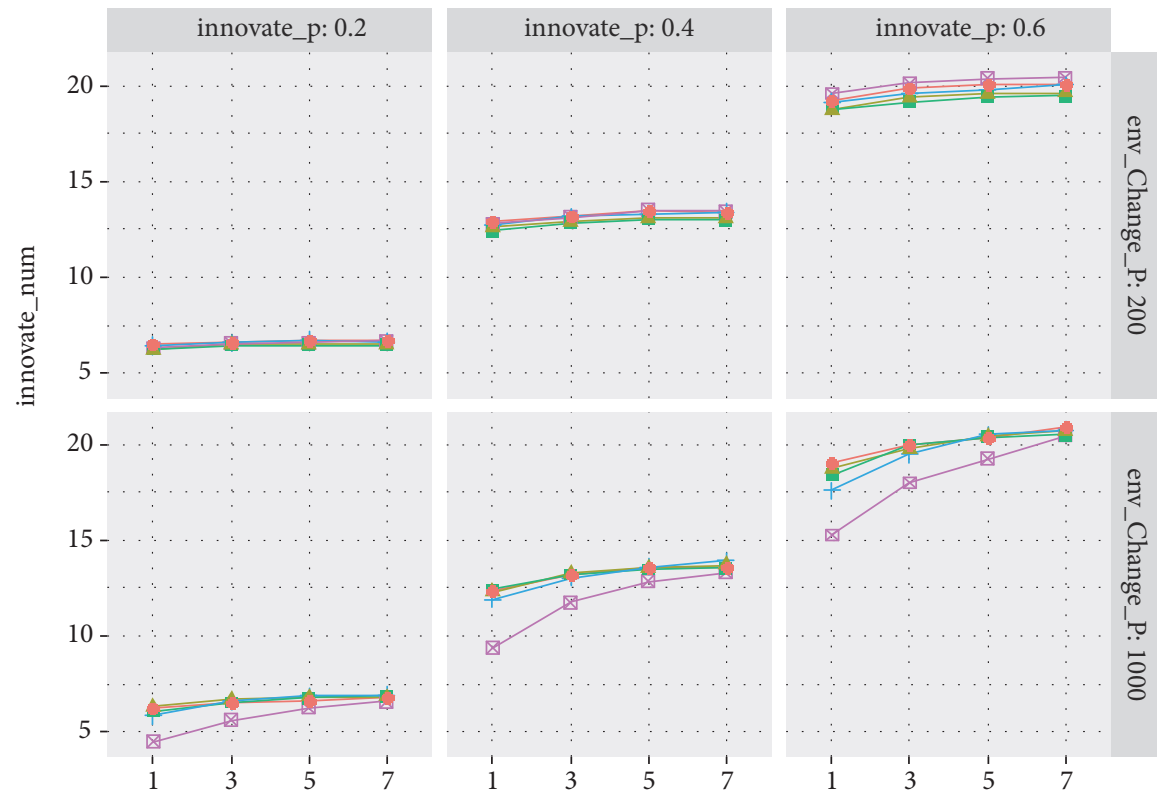

error_p

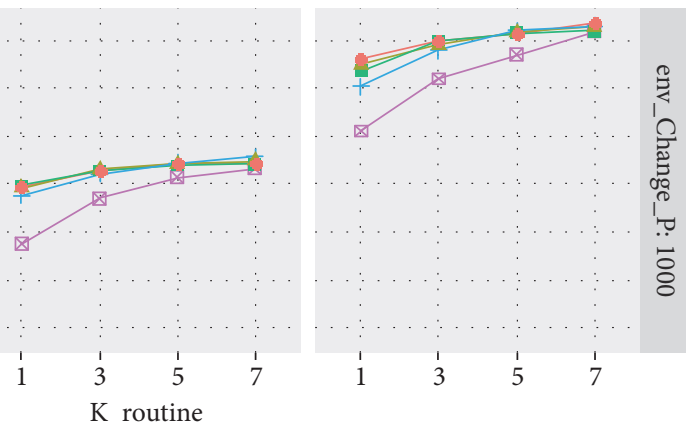

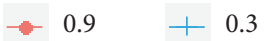
- 0.7 - 0.1
$-0.5$

(b)

Figure 9: Continued. 


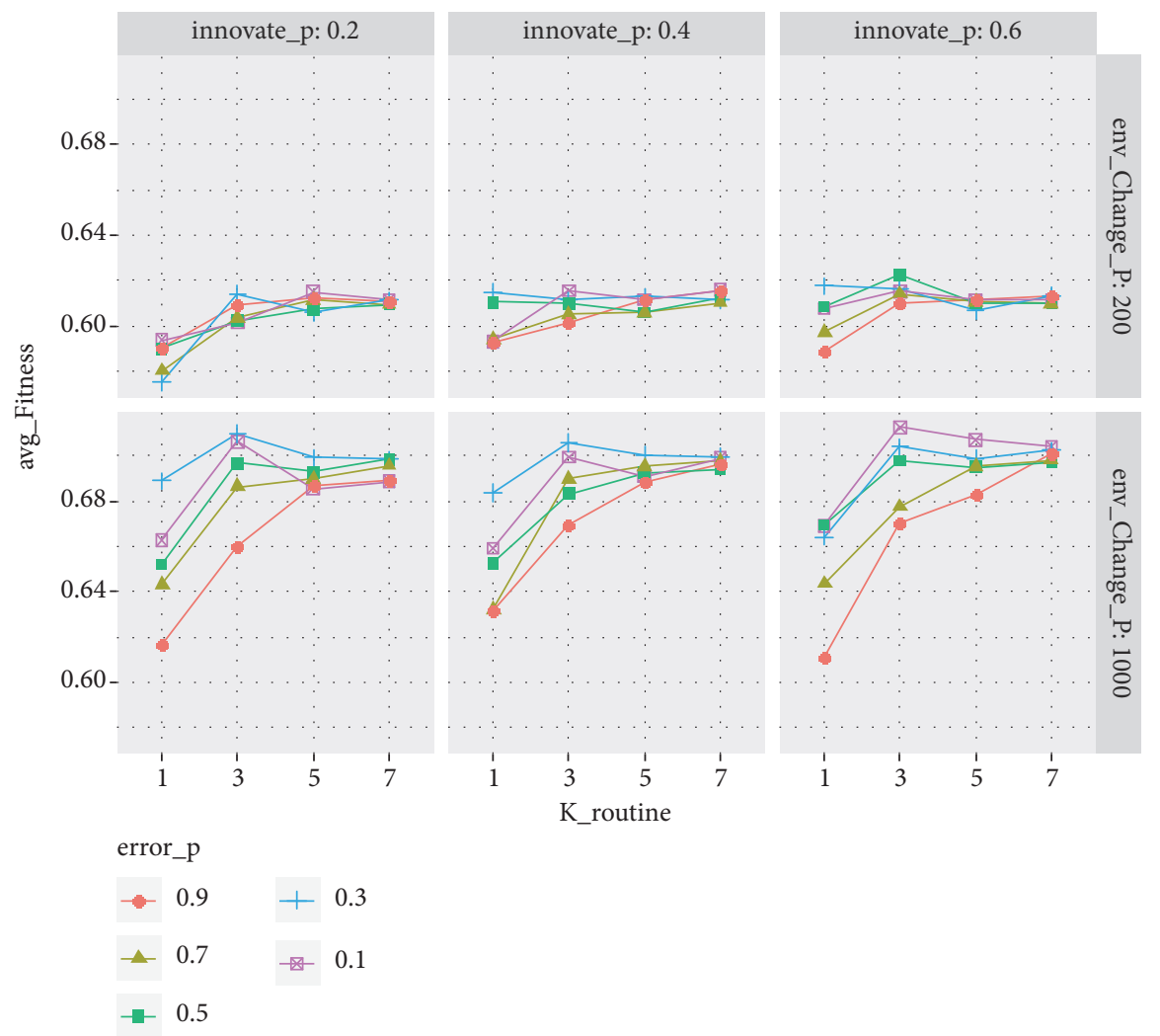

(c)

Figure 9: Simulation results with internally and externally coupled uncertainties (Scenario IV: random_P $=0.5$ and $\left.u n i t \_A C=2\right):(a)$ replicate_num values; (b) innovate_num values; (c) avg_Fitness values.

and innovative activities of unit agents involved, and the uncertainty of environmental changes (more detailed panel regression results can be found in the Appendix).

Our findings showed that in a relatively static environment, a functional replication of routines within a decentralized organization depends on absorptive capacity of unit agents $[18,24]$. Only by an appropriate level of absorptive capacity, each unit can have opportunities to replicate the "best practices" from their neighbors. In this case, an optimal combination of the two different types of networks namely, both the connections between different units and the coupling relations between routines - can be achieved which is beneficial to routine replicating practices that help the organization to adapt to its living environment.

Then, we also found that certain degree of internal variations either from template-duplicating errors or innovative activities of units involved could create opportunities for unit agents to develop "performative drifts" of organizational routines $[32,84]$. While this is beneficial to the adaptiveness of the whole organization, it also comes at operating expenses. However, it is worth noting that a higher level of variations could be harmful to routine replication dynamics $[12,85]$.

Next, we found that although environmental changes require more routine replicating activities, these processes can be detrimental to the whole organization's adaptiveness $[59,86]$. When the environment is dynamic and uncertain, both routine replicating processes and the performance of the organization are sensitive to smaller connecting density values either of the network of unit agents or that of routines.

This implies that increasing connections between different units are not always beneficial to the organization. Managing unit relations always requires balancing costs and benefits [42, 87]. On the other hand, our findings show that the coupling relation of routines is key to cope with the changing pace of the organizational environment [11].

Finally, we found that a certain level of variation from either template-duplicating errors or innovative activities can help organizations to cope with environmental changes better [18]. Here, developing platforms to exploit a certain degree of redundancy and uncertainty from the bottom-up can increase the capacity of an organization to leverage on emergent processes and so cope with turbulent environments better (e.g., [88]). Obviously, this depends on the extent of internal variations, as these could increase operating costs without benefiting organizational performance.

This said our study has important limitations. First, our agent-based model of routine replication dynamics is based on a series of pioneer work (e.g., $[15,17-21])$ that inherited Nelson and Winter's (1982) seminal book [8]. This implies that we focused on routines that are beneficial for organizations' survival and development; i.e., by reconfiguring these routines, creating new ones, and using them consistently 
TABLE 4: Multiple fixed effects panel regressions.

(a) Dep. Variable: steadyTime

\begin{tabular}{|c|c|c|c|c|}
\hline & Scenario I & Scenario II & Scenario III & Scenario IV \\
\hline K_routine & $\begin{array}{c}4.9699^{* * *} \\
(0.9761)\end{array}$ & $\begin{array}{c}20.1570^{* * *} \\
(2.522)\end{array}$ & $\begin{array}{c}7.8355^{* * *} \\
(0.3780)\end{array}$ & $\begin{array}{c}5.3655^{* * *} \\
(0.2659)\end{array}$ \\
\hline random_p & $\begin{array}{c}599.1148^{* * *} \\
(7.7170)\end{array}$ & & $\begin{array}{c}131.6095^{* * *} \\
(2.9886)\end{array}$ & \\
\hline unit_AC & $\begin{array}{c}395.3063^{* * *} \\
(2.6732)\end{array}$ & & $\begin{array}{c}128.1644^{* * *} \\
(1.0353)\end{array}$ & \\
\hline error_p & & $\begin{array}{c}1450.3890^{* * *} \\
(23.913)\end{array}$ & & $\begin{array}{c}35.8058^{* * *} \\
(2.1019)\end{array}$ \\
\hline innovate_p & & $\begin{array}{c}2994.8650^{* * *} \\
\quad(33.1511)\end{array}$ & & $\begin{array}{c}19.0925^{* * *} \\
(3.6406)\end{array}$ \\
\hline$e n v_{-} C h a n g e_{-} P$ & & & $\begin{array}{c}-0.7774^{* * *} \\
(0.0021) \\
\end{array}$ & $\begin{array}{c}-0.0682^{* * *} \\
(0.0015) \\
\end{array}$ \\
\hline R-squared & 338.1 & 617.8 & 149.3 & 105 \\
\hline F-statistic & 9307 & 11850 & 38260 & 707.6 \\
\hline Degree of freedom & 3 and 23996 & 3 and 11996 & 4 and 31195 & 4 and 31195 \\
\hline p-value & 0.0000 & 0.0000 & 0.0000 & 0.0000 \\
\hline
\end{tabular}

(b) Dep. Variable: replicate_num

\begin{tabular}{|c|c|c|c|c|}
\hline & Scenario I & Scenario II & Scenario III & Scenario IV \\
\hline K_routine & $\begin{array}{c}4.3765^{* * *} \\
(0.1269)\end{array}$ & $\begin{array}{c}6.0665^{* * *} \\
(0.2819)\end{array}$ & $\begin{array}{c}2.5261^{* * *} \\
(0.1033)\end{array}$ & $\begin{array}{c}3.4398^{* * *} \\
(0.0580)\end{array}$ \\
\hline random_p & $\begin{array}{c}108.3740^{* * *} \\
\quad(1.0034)\end{array}$ & & $\begin{array}{c}94.7629^{* * *} \\
(0.8164)\end{array}$ & \\
\hline unit_AC & $\begin{array}{c}81.4350^{* * *} \\
(0.3476)\end{array}$ & & $\begin{array}{c}82.5934^{* * *} \\
(0.2828)\end{array}$ & \\
\hline error_p & & $\begin{array}{c}142.4755^{* * *} \\
(2.6726)\end{array}$ & & $\begin{array}{c}83.8480^{* * *} \\
(0.4583)\end{array}$ \\
\hline innovate $p$ & & $\begin{array}{c}80.8739^{* * * *} \\
(3.7051)\end{array}$ & & $\begin{array}{c}5.4678^{* * *} \\
(0.7938)\end{array}$ \\
\hline$e n v_{-} C h a n g e_{-} P$ & & & $\begin{array}{c}0.0101^{* * *} \\
(0.0006)\end{array}$ & $\begin{array}{c}-0.0022^{* * *} \\
(0.0003)\end{array}$ \\
\hline R-squared & 44.0 & 69.1 & 40.8 & 22.9 \\
\hline F-statistic & 22580 & 3159 & 24910 & 9271 \\
\hline Degree of freedom & 3 and 23996 & 3 and 11996 & 4 and 31195 & 4 and 31195 \\
\hline $\mathrm{p}$-value & 0.0000 & 0.0000 & 0.0000 & 0.0000 \\
\hline
\end{tabular}

(c) Dep. Variable: innovate_num

\begin{tabular}{|c|c|c|c|c|}
\hline & Scenario I & Scenario II & Scenario III & Scenario IV \\
\hline K_routine & & $\begin{array}{r}0.2416^{* * *} \\
(0.0086)\end{array}$ & & $\begin{array}{c}0.2041^{* * *} \\
(0.0038)\end{array}$ \\
\hline \multicolumn{5}{|l|}{ random_p } \\
\hline \multicolumn{5}{|l|}{ unit_AC } \\
\hline error_p & & $\begin{array}{r}1.6531^{* * *} \\
(0.0815)\end{array}$ & & $\begin{array}{c}0.6223^{* * *} \\
(0.0299)\end{array}$ \\
\hline innovate_p & & $\begin{array}{c}30.4683^{* * *} \\
(0.1129)\end{array}$ & & $\begin{array}{c}32.7830^{* * *} \\
(0.0517)\end{array}$ \\
\hline
\end{tabular}


(c) Continued.

\begin{tabular}{|c|c|c|c|c|}
\hline & Scenario I & Scenario II & Scenario III & Scenario IV \\
\hline$e n v_{-} C h a n g e \_P$ & & & & $\begin{array}{c}-0.0001^{* * *} \\
(0.0000)\end{array}$ \\
\hline R-squared & & 2.1 & & 1.5 \\
\hline F-statistic & & 49780 & & 10120 \\
\hline Degree of freedom & & 3 and 11996 & & 4 and 31195 \\
\hline $\mathrm{p}$-value & & 0.0000 & & 0.0000 \\
\hline
\end{tabular}

Significance codes: ${ }^{* * *} p<0.001 ;{ }^{* *} p<0.01 ;{ }^{*} p<0.05 ;{ }^{*} p<0.1$.

(d) Dep. Variable: avg_Fitness

\begin{tabular}{|c|c|c|c|c|}
\hline & Scenario I & Scenario II & Scenario III & Scenario IV \\
\hline K_routine & $\begin{array}{c}0.0012^{* * *} \\
(0.0003)\end{array}$ & $\begin{array}{c}0.0060^{* * *} \\
(0.0004)\end{array}$ & $\begin{array}{c}0.0032^{* * *} \\
(0.0002)\end{array}$ & $\begin{array}{c}0.0046^{* * *} \\
(0.0002)\end{array}$ \\
\hline random_p & $\begin{array}{c}0.0839^{* * *} \\
(0.0021)\end{array}$ & & $\begin{array}{c}0.0254^{* * *} \\
(0.0019)\end{array}$ & \\
\hline unit_AC & $\begin{array}{c}0.0626^{* * *} \\
(0.0007)\end{array}$ & & $\begin{array}{r}0.0288^{* * *} \\
(0.0007)\end{array}$ & \\
\hline error_p & & $\begin{array}{c}0.0473^{* * *} \\
(0.0042)\end{array}$ & & $\begin{array}{c}-0.0188^{* * *} \\
(0.0019)\end{array}$ \\
\hline innovate_p & & $\begin{array}{c}0.2273^{* * *} \\
(0.0059)\end{array}$ & $\begin{array}{c}0.0001^{* * *} \\
(0.0000)\end{array}$ & $\begin{array}{c}0.0124^{* * *} \\
(0.0034)\end{array}$ \\
\hline$e n v \_C h a n g e \_P$ & & & & $\begin{array}{c}0.0001^{* * *} \\
(0.0000)\end{array}$ \\
\hline R-squared & 0.09 & 0.11 & 0.10 & 0.10 \\
\hline F-statistic & 2994 & 1426 & 633.3 & 1310 \\
\hline Degree of freedom & 3 and 23996 & 3 and 11996 & 4 and 31195 & 4 and 31195 \\
\hline $\mathrm{p}$-value & 0.0000 & 0.0000 & 0.0000 & 0.0000 \\
\hline
\end{tabular}

Significance codes: ${ }^{* * *} p<0.001 ;{ }^{* *} p<0.01 ;{ }^{*} p<.05 ;{ }^{*} p<0.1$.

across organizational sites, organizations can adapt to a changing environment and obtain a high-level of performance [47-49]. However, "defensive routines" as mentioned in [5-7] are beyond the scope of our discussion. Second, we only limited our concentration on the network characteristic of distributed units within a decentralized organization - i.e., the organization within which activities (particularly those regarding decision-making) are distributed or delegated away from a central, authoritative leadership - rather than considering factors such as leadership and culture $[73,80]$. These factors are, of course, very crucial ones for organization studies, and we would discuss their effects on routine dynamics in detail in another forthcoming paper. Third, the templatebased replication of routines between different units is often linked with some kinds of artifacts - i.e., "physical manifestations" of the performance of organizational routines [89]. These artifacts play an important role in routine dynamics $[20,90,91]$. In this sense, template-duplicating activities can incorporate very complicated interactions between human actors and their material contexts, which are channelled by relating artifacts. Investigating the role of different kinds of artifacts in routine replicating dynamics could complement and enrich our findings. Last but not least, our agent-based model is only a typification and so should be verified with context-specific details $[92,93]$.

\section{Appendix}

\section{Panel Regression Results}

In order to evaluate if the fixed was preferred to the random coefficient panel regressions, we performed a series of Hausman tests using the R package "plm" [94], as shown in Table 4. These tests had significant results with a $p$-value $<0.01$, which indicated that the fixed effects were better fit to the data.

\section{Data Availability}

No empirical data were involved to support this study. We only got input settings of the simulation based on a series of simple specific scenarios testing exercises and repeated "trialand-error" experiments. For each scenario mentioned in the paper, we repeated the simulation running for hundreds of times according to the power analysis results as shown in Table 3 and discussed the simulation results in detail. All the simulation outputs data used in this paper are available from the corresponding author upon request. The NetLogo code 
is available at openABM Archive https://www.comses.net/ codebase-release/459505f3-45be-4316-bb61-91233a04e332/.

\section{Conflicts of Interest}

The authors declare that there are no conflicts of interest regarding the publication of this paper.

\section{Acknowledgments}

This research has been supported by Shandong Provincial Natural Science Foundation (under Grant Nos. ZR2016GB06 and ZR2016GQ07), Shandong Technology and Business University's (SDTBU) Doctoral Foundation (under Grant No. BS201606), and National Natural Science Foundation of China (under Grant No. 71501113).

\section{References}

[1] M. S. Feldman and B. T. Pentland, "Reconceptualizing organizational routines as a source of flexibility and change," Administrative Science Quarterly, vol. 48, no. 1, pp. 94-118, 2003.

[2] M. C. Becker, "The past, present and future of organizational routines: Introduction to the Handbook of Organizational Routines," in The Handbook of Organizational Routines, M. C. Becker and N. Lazaric, Eds., pp. 3-14, Edward Elgar, Cheltenham UK, 2008.

[3] C. Argyris, "Reinforcing organizational defensive routines: An unintended human resources activity," Human Resource Management, vol. 25, no. 4, pp. 541-555, 1986.

[4] C. Argyris, Overcoming organizational defenses efensesng organizational defeensive ro, Allyn and Bacon, Boston, Mass, USA, 1990.

[5] T. Riley, E. Cudney, and S. Long, "A comparative analysis of defensive routines in engineering managers versus nonengineering managers," EMJ-Engineering Management Journal, vol. 25, no. 4, pp. 44-51, 2013.

[6] Y. Yang, D. Secchi, and F. Homberg, "Are organisational defensive routines harmful to the relationship between personality and organisational learning?" Journal of Business Research, vol. 85, pp. 155-164, 2018.

[7] Y. Yang, D. Secchi, and F. Homberg, "Triggers and damages of organizational defensive routines," Problemy ZarzadzaniaManagement Issues, vol. 16, no. 4, 2018.

[8] R. Nelson and S. G. Winter, An Evolutionary Theory of Economic Change, Harvard University Press, Cambridge, Mass, USA, 1982.

[9] M. D. Cohen and P. Bacdayan, "Organizational Routines Are Stored as Procedural Memory: Evidence from a Laboratory Study," Organization Science, vol. 5, no. 4, pp. 554-568, 1994.

[10] B. T. Pentland, T. Hærem, and D. Hillison, "The (N)everchanging world: Stability and change in organizational routines," Organization Science, vol. 22, no. 6, pp. 1369-1383, 2011.

[11] M. S. Feldman, B. T. Pentland, L. D’Adderio, and N. Lazaric, "Beyond Routines as Things: Introduction to the Special Issue on Routine Dynamics," Organization Science, vol. 27, no. 3, pp. 505-513, 2016.

[12] M. C. Becker, "Organizational routines: A review of the literature," Industrial and Corporate Change, vol. 13, no. 4, pp. 643$677,2004$.
[13] T. Felin and N. J. Foss, "Organizational routines and capabilities: Historical drift and a course-correction toward microfoundations," Scandinavian Journal of Management, vol. 25, no. 2, pp. 157-167, 2009.

[14] C. Salvato and C. Rerup, "Beyond collective entities: Multilevel research on organizational routines and capabilities," Journal of Management, vol. 37, no. 2, pp. 468-490, 2011.

[15] S. G. Winter and G. Szulanski, "Replication as Strategy," Organization Science, vol. 12, no. 6, pp. 730-743, 2001.

[16] C. E. Helfat and M. A. Peteraf, "The dynamic resource-based view: Capability lifecycles," Strategic Management Journal, vol. 24, no. 10, pp. 997-1010, 2003.

[17] G. M. Hodgson, “The nature and replication of routines," in Organizational Routines: Advancing Empirical Research, M. C. Becker and N. Lazaric, Eds., pp. 26-46, Edward Elgar, Cheltenham UK, 2009.

[18] M. Friesl and J. Larty, "Replication of Routines in Organizations: Existing Literature and New Perspectives," International Journal of Management Reviews, vol. 15, no. 1, pp. 106-122, 2013.

[19] R. J. Jensen, Essays on the Replication of Organizational Routines: The Effect of Templates on Knowledge Transfer, the Mechanisms Underlying Knowledge Transfer Methods, and Variation Through Replication [Doctoral, thesis], University of Pennsylvania, 2006.

[20] L. D’Adderio, “The replication dilemma unravelled: How organizations enact multiple goals in routine transfer," Organization Science, vol. 25, no. 5, pp. 1325-1350, 2014.

[21] G. Szulanski and R. J. Jensen, "Overcoming stickiness: An empirical investigation of the role of the template in the replication of organizational routines," Managerial and Decision Economics, vol. 25, no. 6-7, pp. 347-363, 2004.

[22] J. A. Howard-Grenville, "The persistence of flexible organizational routines: The role of agency and organizational context," Organization Science, vol. 16, no. 6, pp. 618-636, 2005.

[23] S. G. Winter and G. Szulanski, "Replication of organizational routines: Conceptualizing the exploitation of knowledge assets," in The Strategic Management of Intellectual Capital and Organizational Knowledge, C. W. Choo and N. Bontis, Eds., pp. 207221, 2002.

[24] G. Szulanski, "Exploring internal stickiness: Impediments to the transfer of best practice within the firm," Strategic Management Journal, vol. 17, no. WINTER, pp. 27-43, 1996.

[25] L. Argote and P. Ingram, "Knowledge transfer: a basis for competitive advantage in firms," Organizational Behavior and Human Decision Processes, vol. 82, no. 1, pp. 150-169, 2000.

[26] M. C. Becker and N. Lazaric, "The Influence of Knowledge in the Replication of Routines," in Économie appliquée: archives de l'Institut de science économique appliquée, vol. 3, pp. 65-94, Institut des sciences mathématiques et économiques appliquées - ISMEA, 2003.

[27] W. Tsai, "Knowledge transfer in intraorganizational networks: effects of network position and absorptive capacity on business unit innovation and performance," Academy of Management Journal (AMJ), vol. 44, no. 5, pp. 996-1004, 2001.

[28] R. Reagans and B. McEvily, "Network structure and knowledge transfer: The effects of cohesion and range," Administrative Science Quarterly, vol. 48, no. 2, pp. 240-356, 2003.

[29] J. Mu, F. Tang, and D. L. MacLachlan, "Absorptive and disseminative capacity: Knowledge transfer in intra-organization networks," Expert Systems with Applications, vol. 37, no. 1, pp. 31-38, 2010. 
[30] S. G. Winter, "Capabilities: Their Origins and Ancestry," Journal of Management Studies, vol. 49, no. 8, pp. 1402-1406, 2012.

[31] M. Friesl and J. Larty, Replication of routines and capabilities: From knowledge transfer to replication as a social practice, Entrepreneurship and Enterprise Development Working Paper Series, Institute for Entrepreneurship and Enterprise Development, Lancaster University, 2010.

[32] W. J. Orlikowski, "Knowing in practice: Enacting a collective capability in distributed organizing," Organization Science, vol. 13, no. 3, pp. 249-273, 2002.

[33] H. Tsoukas and C. Knudsen, "The conduct of strategy research," in Handbook of Strategy Management, A. Pettigrew, H. Thomas, and R. Whittington, Eds., pp. 411-435, 2006.

[34] S. Bertels, J. Howard-Grenville, and S. Pek, "Cultural Molding, Shielding, and Shoring at Oilco: The Role of Culture in the Integration of Routines," Organization Science, vol. 27, no. 3, pp. 573-593, 2016.

[35] J. P. Birnholtz, M. D. Cohen, and S. V. Hoch, "Organizational character: On the regeneration of camp poplar grove," Organization Science, vol. 18, no. 2, pp. 315-332, 2007.

[36] D. X. Peng, R. G. Schroeder, and R. Shah, "Linking routines to operations capabilities: A new perspective," Journal of Operations Management, vol. 26, no. 6, pp. 730-748, 2008.

[37] A. Parmigiani and J. Howard-Grenville, "Routines revisited: Exploring the capabilities and practice perspectives," Academy of Management Annals, vol. 5, no. 1, pp. 413-453, 2011.

[38] C. Salvato and C. Rerup, "Routine Regulation: Balancing Conflicting Goals in Organizational Routines,", Administrative Science Quarterly, vol. 63, no. 1, pp. 170-209, 2018.

[39] W. Kremser and G. Schreyögg, "The Dynamics of Interrelated Routines: Introducing the Cluster Level," Organization Science, vol. 27, no. 3, pp. 698-721, 2016.

[40] D. Dönmez, G. Grote, and S. Brusoni, "Routine interdependencies as a source of stability and flexibility. A study of agile software development teams," Information and Organization, vol. 26, no. 3, pp. 63-83, 2016.

[41] M. S. Feldman and A. Rafaeli, "Organizational routines as sources of connections and understandings," Journal of Management Studies, vol. 39, no. 3, pp. 309-331, 2002.

[42] S. F. Turner and V. Rindova, "A balancing act: How organizations pursue consistency in routine functioning in the face of ongoing change," Organization Science, vol. 23, no. 1, pp. 24-46, 2012.

[43] S. A. Kauffman, The Origins of Order: Self-Organization and Selection in Evolution, Oxford University Press, Oxford, UK, 1993.

[44] N. Gilbert, Agent-Based Models, Sage Publications, Guildford, UK, 2008.

[45] K. D. Miller, "Agent-Based Modeling and Organization Studies: A critical realist perspective," Organization Studies, vol. 36, no. 2, pp. 175-196, 2015.

[46] U. Wilensky and W. Rand, An Introduction to Agent-based Modeling: Modeling Natural, Social, and Engineered Complex Systems with NetLogo, The MIT Press, 2015.

[47] M. Zollo and S. G. Winter, "Deliberate learning and the evolution of dynamic capabilities," Organization Science, vol. 13, no. 3, pp. 339-351, 2002.

[48] D. J. Teece, G. Pisano, and A. Shuen, "Dynamic capabilities and strategic management," Strategic Management Journal, vol. 18, no. 7, pp. 509-533, 1997.
[49] D. J. Teece, "Explicating dynamic capabilities: The nature and microfoundations of (sustainable) enterprise performance," Strategic Management Journal, vol. 28, no. 13, pp. 1319-1350, 2007.

[50] G. M. Hodgson, "The mystery of the routine the darwinian destiny of an evolutionary theory of economic change," Revue Économique, vol. 54, no. 2, pp. 355-384, 2003.

[51] J. W. Rivkin, "Reproducing Knowledge: Replication Without Imitation at Moderate Complexity," Organization Science, vol. 12, no. 3, pp. 274-293, 2001.

[52] S. G. Winter, G. Szulanski, D. Ringov, and R. J. Jensen, "Reproducing knowledge: Inaccurate replication and failure in franchise organizations," Organization Science, vol. 23, no. 3, pp. 672-685, 2012.

[53] G. Szulanski, "The process of knowledge transfer: a diachronic analysis of stickiness," Organizational Behavior and Human Decision Processes, vol. 82, no. 1, pp. 9-27, 2000.

[54] T. Kostova and K. Roth, "Adoption of an organizational practice by subsidiaries of multinational corporations: Institutional and relational effects," Academy of Management Journal (AMJ), vol. 45, no. 1, pp. 215-233, 2002.

[55] R. J. Jensen and G. Szulanski, "Template use and the effectiveness of knowledge transfer," Management Science, vol. 53, no. 11, pp. 1716-1730, 2007.

[56] I. Maurer, V. Bartsch, and M. Ebers, “The value of intraorganizational social capital: How it fosters knowledge transfer, innovation performance, and growth," Organization Studies, vol. 32, no. 2, pp. 157-185, 2011.

[57] C. A. Maritan and T. H. Brush, "Heterogeneity and transferring practices: Implementing flow manufacturing in multiple plants," Strategic Management Journal, vol. 24, no. 10, pp. 945959, 2003.

[58] G. Dosi, R. R. Nelson, and S. Winter, The Nature and Dynamics of Organizational Capabilities, Oxford University Press, New York, NY, USA, 2001.

[59] K. M. Eisenhardt and J. A. Martin, "Dynamic capabilities: What are they?" Strategic Management Journal, vol. 21, no. 10-11, pp. 1105-1121, 2000.

[60] K. Sele and S. Grand, "Unpacking the Dynamics of Ecologies of Routines: Mediators and Their Generative Effects in Routine Interactions," Organization Science, vol. 27, no. 3, pp. 722-738, 2016.

[61] M. A. Peteraf, “The cornerstones of competitive advantage: A resource-based view," Strategic Management Journal, vol. 14, no. 3, pp. 179-191, 1993.

[62] P. Milgrom and J. Roberts, "Complementarities and fit strategy, structure, and organizational change in manufacturing," Journal of Accounting and Economics, vol. 19, no. 2-3, pp. 179-208, 1995.

[63] C. Salvato, "Capabilities unveiled: The role of ordinary activities in the evolution of product development processes," Organization Science, vol. 20, no. 2, pp. 384-409, 2009.

[64] S. Wright, "The roles of mutation, inbreeding, crossbreeding," in Proceedings of the Sixth International Congress on Genetics, pp. 355-366, 1932.

[65] D. A. Levinthal, “Adaptation on rugged landscapes," Management Science, vol. 43, no. 7, pp. 934-950, 1997.

[66] K. Frenken, "A fitness landscape approach to technological complexity, modularity, and vertical disintegration," Structural Change and Economic Dynamics, vol. 17, no. 3, pp. 288-305, 2006. 
[67] G. Gavetti, D. A. Levinthal, and J. W. Rivkin, "Strategy making in novel and complex worlds: The power of analogy," Strategic Management Journal, vol. 26, no. 8, pp. 691-712, 2005.

[68] M. Ganco and G. Hoetker, "NK modeling methodology in the strategy literature: Bounded search on a rugged landscape," in Research Methodology in Strategy and Management, D. D. Bergh and D. J. Ketchen, Eds., pp. 237-268, Emerald Group Publishing, 2009.

[69] G. Dosi, M. Faillo, L. Marengo, and D. Moschella, “"Modeling routines and organizational learning: A discussion of the stateof-the-art,” LEM Working Paper 2011/04, 2011.

[70] M. J. Ventresca and W. N. Kaghan, "Routines, "going concerns" and innovation: Towards an evolutionary economic sociology," in The Handbook of Organizational Routines, M. C. Becker, Ed., pp. 52-86, Edward Elgar, 2008.

[71] S. Yi, T. Knudsen, and M. C. Becker, "Inertia in Routines: A Hidden Source of Organizational Variation," Organization Science, vol. 27, no. 3, pp. 782-800, 2016.

[72] S. K. Ethiraj and D. Levinthal, "Modularity and innovation in complex systems," Management Science, vol. 50, no. 2, pp. 159173, 2004.

[73] N. Lazaric and A. Raybaut, "Knowledge, hierarchy and the selection of routines: An interpretative model with group interactions," Journal of Evolutionary Economics, vol. 15, no. 4, pp. 393-421, 2005.

[74] P. Erdös and A. Rényi, "On the evolution of random graphs," Publications of the Mathematical Institute of the Hungarian Academy of Sciences, vol. 5, pp. 17-61, 1960.

[75] H. Simon, Administrative Behavior, Free Press, New York, NY, USA, 1945.

[76] C. G. Gilbert, "Unbundling the structure of inertia: Resource versus routine rigidity," Academy of Management Journal (AMJ), vol. 48, no. 5, pp. 741-763, 2005.

[77] C. Camisón and B. Forés, "Knowledge absorptive capacity: New insights for its conceptualization and measurement," Journal of Business Research, vol. 63, no. 7, pp. 707-715, 2010.

[78] A. Y. Lewin, S. Massini, and C. Peeters, "Microfoundations of internal and external absorptive capacity routines," Organization Science, vol. 22, no. 1, pp. 81-98, 2011.

[79] M. J. North and C. M. Macal, Managing Business Complexity: Discovering Strategic Solutions with Agent-based Modeling and Simulation, Oxford University Press, New York, NY, USA, 2007.

[80] J. Goldstein, J. K. Hazy, and B. B. Lichtenstein, Complexity and the Nexus of Leadership, Palgrave Macmillan US, New York, 2010.

[81] D. Secchi and R. Seri, "Controlling for false negatives in agentbased models: a review of power analysis in organizational research," Computational and Mathematical Organization Theory, vol. 23, no. 1, pp. 94-121, 2017.

[82] R. Seri and D. Secchi, "How Many Times Should One Run a Computational Simulation?" in Simulating Social Complexity, B. Edmonds and R. Meyer, Eds., Understanding Complex Systems, pp. 229-251, Springer International Publishing, Cham, Switzerland, 2017.

[83] R. Gore, S. Diallo, C. Lynch, and J. Padilla, "Augmenting Bottom-up Metamodels with Predicates," Journal of Artificial Societies and Social Simulation, vol. 20, no. 1, 2017.

[84] J. Balogun and G. Johnson, "From intended strategies to unintended outcomes: The impact of change recipient sensemaking," Organization Studies, vol. 26, no. 11, pp. 1573-1601, 2005.
[85] K. E. Weick, "The Vulnerable System: An Analysis of the Tenerife Air Disaster," Journal of Management, vol. 16, no. 3, pp. 571-593, 1990.

[86] B. W. Wirtz, A. Mathieu, and O. Schilke, "Strategy in HighVelocity Environments," Long Range Planning, vol. 40, no. 3, pp. 295-313, 2007.

[87] D. Gao, X. Deng, Q. Zhao, H. Zhou, and B. Bai, "Multi-Agent Based Simulation of Organizational Routines on Complex Networks," Journal of Artificial Societies and Social Simulation, vol. 18, no. 3, 2015.

[88] J. K. Hazy and M. Uhl-Bien, "Towards operationalizing complexity leadership: How generative, administrative and community-building leadership practices enact organizational outcomes," Leadership, vol. 11, no. 1, pp. 79-104, 2015.

[89] B. T. Pentland and M. S. Feldman, "Designing routines: On the folly of designing artifacts, while hoping for patterns of action," Information and Organization, vol. 18, no. 4, pp. 235-250, 2008.

[90] L. Dadderio, "Artifacts at the centre of routines: Performing the material turn in routines theory," Journal of Institutional Economics, vol. 7, no. 2, pp. 197-230, 2011.

[91] D. Gao, F. Squazzoni, and X. Deng, “The role of cognitive artifacts in organizational routine dynamics: an agent-based model," Computational and Mathematical Organization Theory, pp. 1-27, 2018.

[92] R. Boero and F. Squazzoni, "Does empirical embeddedness matter? Methodological issues on agent-based models for analytical social science," Journal of Artificial Societies and Social Simulation, vol. 8, no. 4, 2005.

[93] R. Tobias and H.-J. Mosler, "Optimizing Campaigns for Changing Routine Behaviors by Using an Empirically Calibrated Microsimulation Model," Social Science Computer Review, vol. 35, no. 2, pp. 184-202, 2017.

[94] Y. Croissant and G. Millo, "Panel data econometrics in R: The plm package," Journal of Statistical Software, vol. 27, no. 2, pp. $1-43,2008$. 


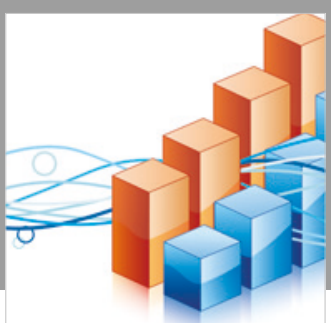

Advances in

Operations Research

\section{-n-m}
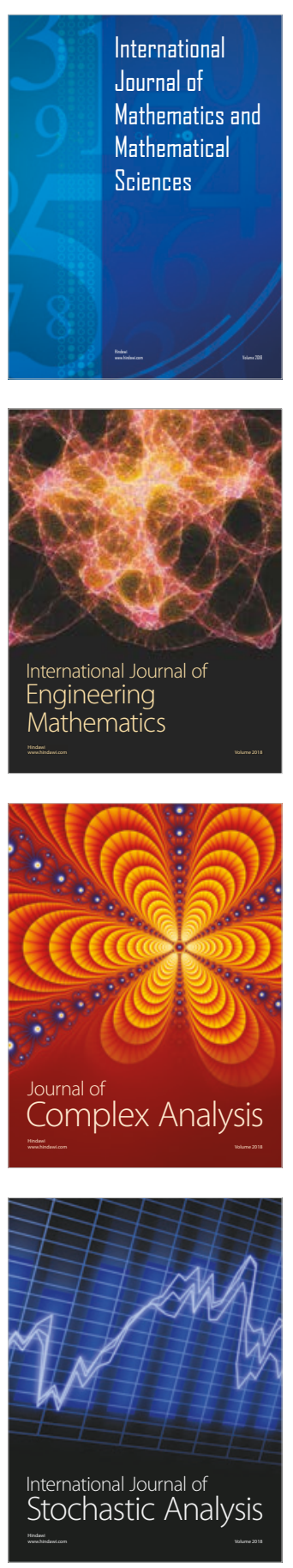
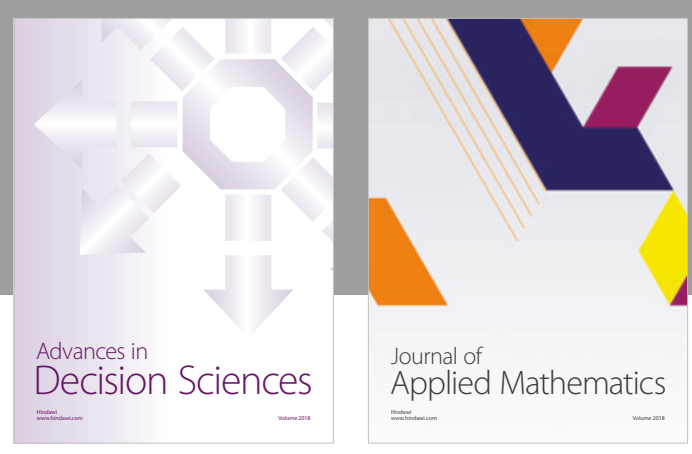

Journal of

Applied Mathematics
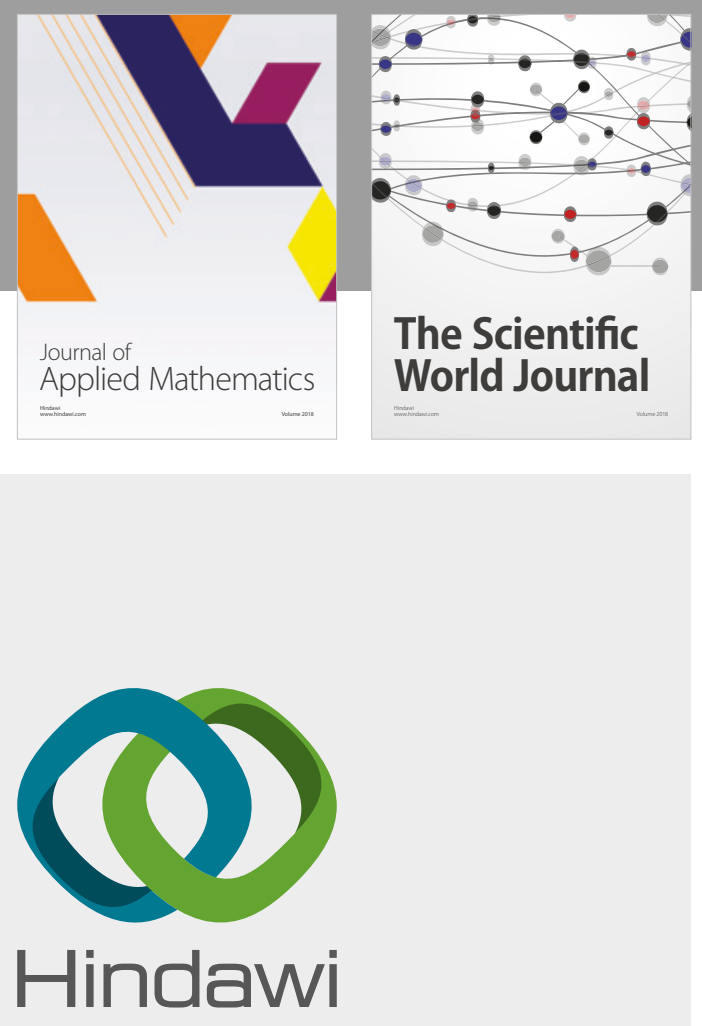

Submit your manuscripts at

www.hindawi.com

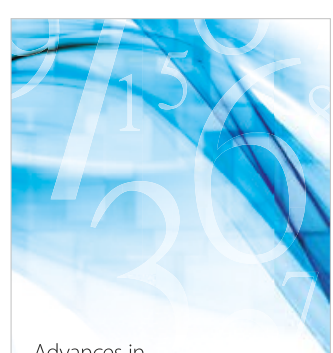

Advances in
Numerical Analysis
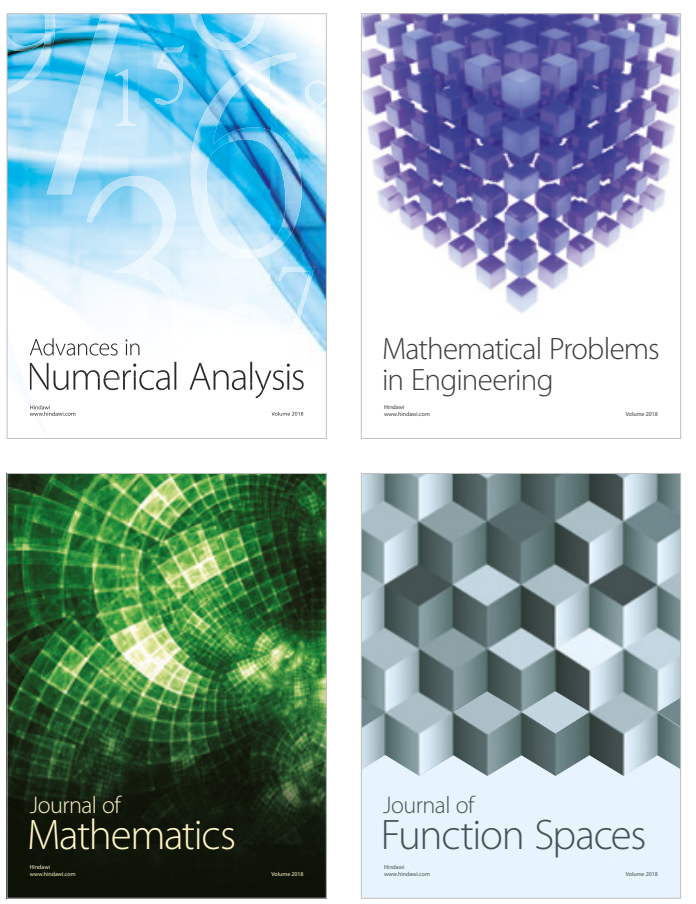

Mathematical Problems in Engineering

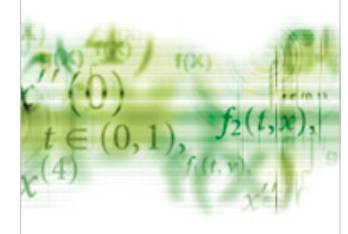

International Journal of

Differential Equations

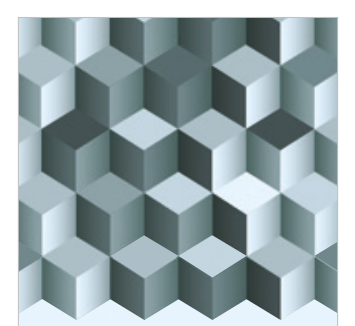

Journal of

Function Spaces
The Scientific

World Journal

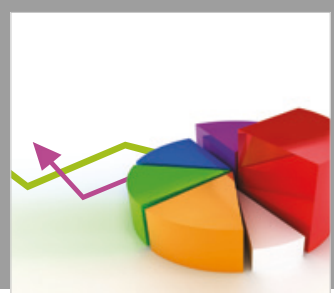

Journal of

Probability and Statistics
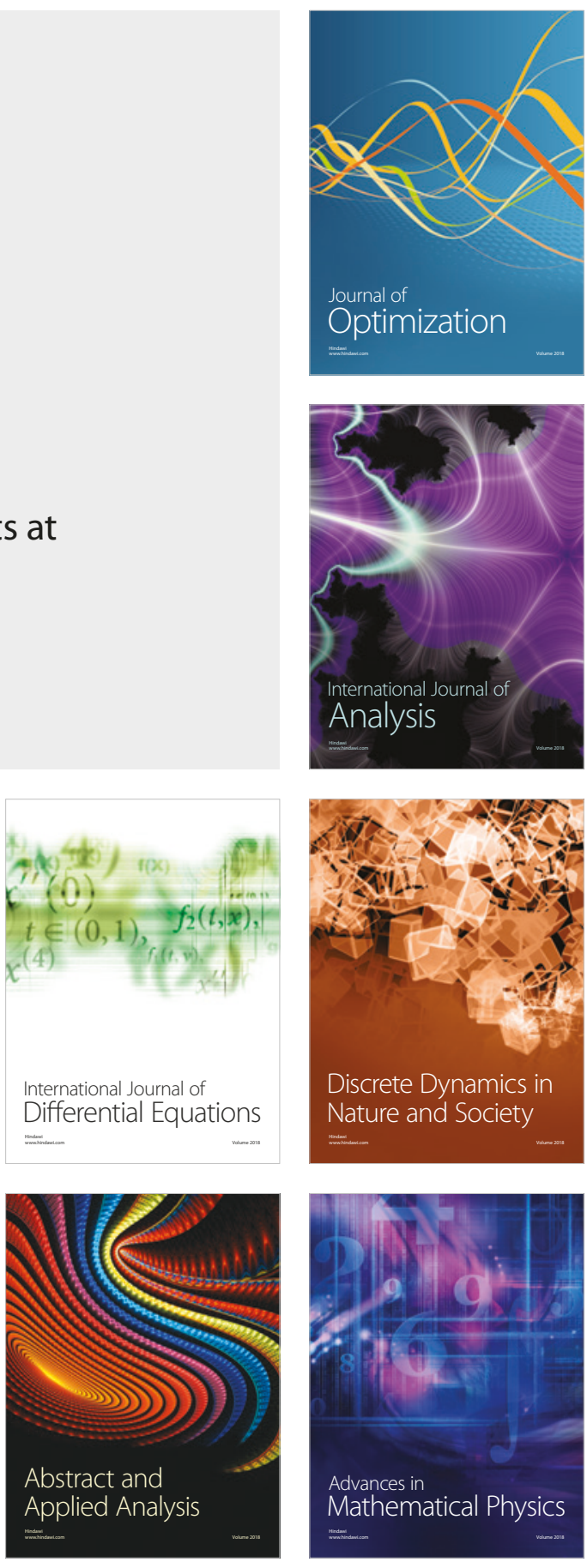